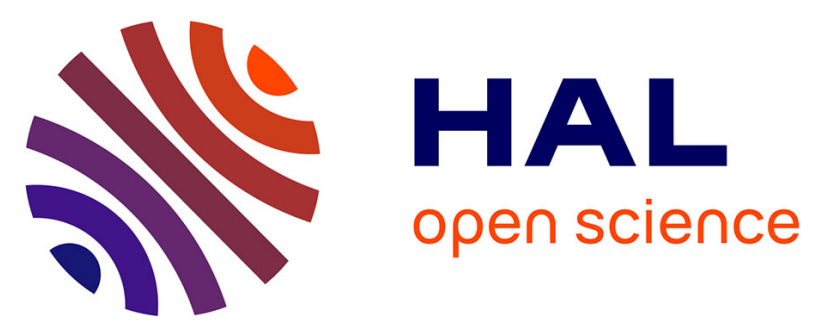

\title{
A neural mass model with direct and indirect excitatory feedback loops: identification of bifurcations and temporal dynamics
}

\author{
Aurélie Garnier, Alexandre Vidal, Clément Huneau, Habib Benali
}

\section{To cite this version:}

Aurélie Garnier, Alexandre Vidal, Clément Huneau, Habib Benali. A neural mass model with direct and indirect excitatory feedback loops: identification of bifurcations and temporal dynamics. Neural Computation, 2015, 27 (2), pp.329-364. 10.1162/NECO_a_00696 . hal-01112146

\section{HAL Id: hal-01112146 \\ https://hal.sorbonne-universite.fr/hal-01112146}

Submitted on 2 Feb 2015

HAL is a multi-disciplinary open access archive for the deposit and dissemination of scientific research documents, whether they are published or not. The documents may come from teaching and research institutions in France or abroad, or from public or private research centers.
L'archive ouverte pluridisciplinaire HAL, est destinée au dépôt et à la diffusion de documents scientifiques de niveau recherche, publiés ou non, émanant des établissements d'enseignement et de recherche français ou étrangers, des laboratoires publics ou privés. 


\title{
A Neural Mass Model with Direct and Indirect Excitatory Feedback Loops: Identification of Bifurcations and Temporal Dynamics
}

\author{
Aurélie Garnier \\ Aurelie.Garnier@lib.upmc.fr \\ Sorbonne Universités, UPMC Univ Paris 06, UMR 7371, UMR_S 1146, Laboratoire \\ d'Imagerie Biomédicale, F-75013, Paris, France; CNRS, UMR 7371, Laboratoire \\ d'Imagerie Biomédicale, F-75013, Paris, France; and INSERM, UMR_S 1146, \\ Laboratoire d'Imagerie Biomédicale, F-75013, Paris, France
}

\author{
Alexandre Vidal \\ alexandre.vidal@univ-evry.fr \\ Université d'Évry-Val-d'Essonne, CNRS UMR 8071, Laboratoire de Mathématiques \\ et Modélisation d'Évry (LaMME), F-91000, Évry, France
}

\section{Clément Huneau}

clement.huneau@inserm.fr

Sorbonne Universités, UPMC Univ Paris 06, UMR 7371, UMR_S 1146, Laboratoire d'Imagerie Biomédicale, F-75013, Paris, France; CNRS, UMR 7371, Laboratoire d'Imagerie Biomédicale, F-75013, Paris, France; INSERM UMR_S 1146, Laboratoire d'Imagerie Biomédicale, F-75013, Paris, France; Université Paris Diderot, Sorbonne Cité, UMR-S1161, F-75010, Paris, France; and INSERM, U1161, F-75010, Paris, France

\section{Habib Benali}

habib.benali@lib.upmc.fr

Sorbonne Universités, UPMC Univ Paris 06, UMR 7371, UMR_S 1146, Laboratoire d'Imagerie Biomédicale, F-75013, Paris, France; CNRS, UMR 7371, Laboratoire d'Imagerie Biomédicale, F-75013, Paris, France; and INSERM UMR_S 1146, Laboratoire d'Imagerie Biomédicale, F-75013, Paris, France

Neural mass modeling is a part of computational neuroscience that was developed to study the general behavior of a neuronal population. This type of mesoscopic model is able to generate output signals that are comparable to experimental data, such as electroencephalograms. Classically, neural mass models consider two interconnected populations: excitatory pyramidal cells and inhibitory interneurons. However, many authors have included an excitatory feedback on the pyramidal cell population. Two distinct approaches have been developed: a direct feedback on the main pyramidal cell population and an indirect feedback via a secondary 
pyramidal cell population. In this letter, we propose a new neural mass model that couples these two approaches. We perform a detailed bifurcation analysis and present a glossary of dynamical behaviors and associated time series. Our study reveals that the model is able to generate particular realistic time series that were never pointed out in either simulated or experimental data. Finally, we aim to evaluate the effect of balance between both excitatory feedbacks on the dynamical behavior of the model. For this purpose, we compute the codimension 2 bifurcation diagrams of the system to establish a map of the repartition of dynamical behaviors in a direct versus indirect feedback parameter space. A perspective of this work is, from a given temporal series, to estimate the parameter value range, especially in terms of direct versus indirect excitatory feedback.

\section{Introduction}

The purpose of computational neurosciences is to develop calculation methods to better understand the complex relationship between brain function and structure. In addition to gaining a better understanding of cognition and its dysfunction, this process enables a transfer of neuroscience knowledge by offering new ways to process information. Developing new computational models and studying their mathematical properties to simulate neuronal population activity, and comparing these simulations with actual data, allows us to improve our understanding of the mechanisms of brain function in humans and other animals.

At the microscopic level, neurons in the brain communicate via spontaneous and rapid variations in membrane potential, action potential, or nerve impulses. Together these electrical phenomena and their related properties have been studied in models of firing neurons, seen as dynamical systems. These models, such as those of Hodgkin and Huxley (1952), enable us to understand and simulate mechanisms that reproduce neuronal behavior in generating action potential, dendritic integration, and axonal propagation. It is also possible to study the overall behavior of a neuronal population using networks of microscopic models (Brunel \& Wang, 2001; Wong \& Wang, 2006; Stefanescu \& Jirsa, 2008) or macroscopic computational models based on the organization of cell interactions. For example, these models allow one to better understand how the balance between neuronal excitation and inhibition affects the genesis of neuronal rhythmic activity.

For this purpose, several approaches such as mean field, neural field, and neural mass models have been developed, as reviewed in Deco, Jirsa, Robinson, Breakspear, and Friston (2008). Mean field models consider the evolution of the membrane potential of a population of statistically similar integrate-and-fire neurons, also called population density or ensemble density (Knight, Manin, \& Sirovich, 1996; Omurtag, Knight, \& Sirovich, 2000). Neural field models represent the activity of specific mean field models 
(a)

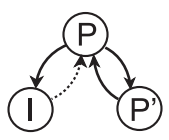

(b)

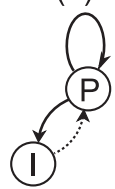

(c)

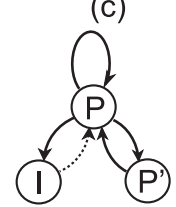

Figure 1: Diagrams of interactions between neuronal populations: indirect (a), direct (b), and double (c) excitatory feedback from pyramidal cells. P: Population of pyramidal neurons. $\mathrm{P}^{\prime}$ : Intermediary population of pyramidal neurons. I: Inhibitory interneuron population. The plain (resp. dashed) arrows represent excitation (resp. inhibition).

with respect to time and space (Jirsa \& Haken, 1996, 1997). Finally, the neural mass approach was originally introduced by Beurle (1956), who studied the propagation of activity waves in a distribution model of a mass of cells by focusing on the density of active neurons. Then Griffith and coauthors used a neural field formalism and introduced a second-order linear differential operator to represent both excitatory and inhibitory interactions between neural cells (Griffith, 1963, 1965). Thereafter, Wilson and Cowan $(1972,1973)$ derived the nonlinear temporal dynamics for spatially localized neuron populations (voxel) driving the average firing rates associated with a two-subpopulation model. Their work popularized neural mass models (NMM) using the ordinary differential equation formalism as an efficient tool for studying the interaction mechanisms at a mesoscopic level. Besides, the NMMs produce output signals, especially local field potentials (LFP), comparable to experimental results such as those produced by electroencephalography (EEG) (Lopes da Silva, Hoeks, Smits, \& Zetterberg, 1974).

NMMs mainly involved the interactions of two subpopulations: the principal excitatory cells and the inhibitory interneurons. For a more realistic modeling, some authors have considered a feedback of each subpopulation on itself. In particular, for the excitatory feedback of principal cells, two approaches have been considered. On one hand, a classic way to model this excitatory feedback involving a direct link from the output of principal cells to their input (see Figure 1b) was proposed by Wilson and Cowan (1973) and used by many authors (Robinson, Rennie, \& Wright, 1997; Liley, Cadush, \& Dafilis, 2002; Molaee-Ardekani, Benquet, Bartolomei, \& Wendling, 2010). On the other hand, Jansen and coworkers proposed an indirect track using an intermediate excitatory population (see Figure 1a) (Jansen \& Rit, 1995; Jansen, Zouridakis, \& Brandt, 1993). Their model is based on a principal population of pyramidal cells, connected to a population of interneurons as well as another pyramidal cell population acting as an intermediary for excitatory feedback. Interactions between these populations are those introduced by Lopes da Silva and coauthors (Lopes da Silva et al., 1974; Lopes da Silva, van Rotterdam, Barts, van Heusden, \& Burr, 1976), and the 
indirect excitatory feedback of pyramidal cells follows the structure studied by Katznelson (1981). Afterward, (Wendling, Bellanger, Bartolomei, \& Chauvel, 2000) applied similar models using indirect excitatory feedback to simulate paroxystic neural activity in the context of partial epilepsies.

Both direct and indirect approaches model the excitatory synaptic interaction between neighbor principal cells (Miles \& Wong, 1986; Wang et al., 2006; Frick, Feldmeyer, Helmstaedter, \& Sakmann, 2008), usually named collateral excitation. Nevertheless, a difference may exist in the range of the local connections considered in these approaches. In practical terms, an indirect feedback induces a delay in the excitatory coupling, which may model a larger neighborhood involved in this feedback. Therefore, direct feedback models produce different outputs that display faster activities than those produced by the indirect feedback models. In fact, the different couplings studied imply specific underlying dynamics of the model and therefore give rise to different panels of behaviors. The emergence of identifiable temporal output features (e.g., subthreshold oscillations, epileptic spikes) can be characterized or predicted by understanding these dynamics.

From the modeling perspective, we cannot privilege one type of feedback over the other since both of these couplings are physiologically relevant and can coexist-a very local one and a more or less distant one. To the best of our knowledge, no computational model simultaneously integrating these types of feedback has been published in the literature to date.

Traditionally NMMs receive an input representing the external action of other neuron population activities from other cortical (or subcortical) units on the main pyramidal cell population. This activity can be a stochastic variable representing a nonspecific background activity (Lopes da Silva et al., 1974, 1976; Jansen \& Rit, 1995; Wendling et al., 2000; Wendling, Bartolomei, Bellanger, \& Chauvel, 2002) or a deterministic input representing a specific activity in another cortical unit (Jansen et al., 1993; Huneau et al., 2013). The outputs are therefore calibrated (e.g., according to the frequency and amplitude) based on the average and variance of this input. In the case of the Jansen-Rit model (Jansen \& Rit, 1995), a codimension 2 bifurcation analysis of the system made it possible to identify the intrinsic dynamical properties to the emergence of epileptic behavior (Touboul, Wendling, Chauvel, \& Faugeras, 2011). In this study, the two parameters of interest are the noise (considered to be a parameter in the study of bifurcations) and the maximum number of synaptic connections. Since this latter parameter $C$ is very difficult to quantify physiologically at the voxel scale, a relevant use of such a model consists in characterizing the changes in the behavior associated with the changes in this parameter value.

In this letter, we propose a model that includes both feedback circuits (see Figure 1c) weighted by coupling gain parameters. Therefore, this new model specifically includes the two previous approaches. We show behaviors, in terms of time series, that this model can produce and the associated 
organizations of the dynamics. Using the codimension 2 bifurcation diagrams according to the input value and the synaptic connection rate, we then analyze the model behavior distribution according to the direct and indirect feedback gains. Finally, we discuss the use of this theoretical approach to identify these gain values based on time series trials.

\section{Neural Mass Model with Double Excitatory Feedback}

Traditionally the neural mass models looked at a pyramidal cell population receiving inhibitory input from a local interneuron population and an excitatory input. Collateral excitation of the pyramidal cell population by its own activity is also relayed by synaptic connections. The firing rate of each population results from integrating the input that it receives via the synaptic connections. Following classical modeling (Lopes da Silva et al., 1974, 1976; Jansen et al., 1993; Jansen \& Rit, 1995), we assume the synaptic integration is linear, which means that the membrane potential of each population is a convolution of the input with the synaptic impulse response. This impulse response, which represents the average postsynaptic potential, is characterized by a rapid rise followed by a slower decay.

2.1 Synaptic Coupling in Neural Mass Models. Two functions have been introduced to represent the transformation of the average pulse density into excitatory and inhibitory postsynaptic potentials, respectively. We use the following functions introduced by Van Rotterdam, Lopes da Silva, Van den Ende, Viergever, and Hermans (1982):

$$
\begin{aligned}
& h_{e}(t)=A a t e^{-a t}, \quad t \geqslant 0, \\
& h_{i}(t)=B b t e^{-b t}, \quad t \geqslant 0 .
\end{aligned}
$$

Functions $h_{e}$ and $h_{i}$, respectively, are the basic solutions of operators $\mathcal{F}_{e}$ and $\mathcal{F}_{i}$ as follows (Touboul et al., 2011):

$$
\begin{aligned}
& \mathcal{F}_{e}\left(h_{e}\right)=\frac{1}{A a} h_{e}^{\prime \prime}(t)-\frac{2}{A} h_{e}^{\prime}(t)-\frac{a}{A} h_{e}(t), \\
& \mathcal{F}_{i}\left(h_{i}\right)=\frac{1}{B b} h_{i}^{\prime \prime}(t)-\frac{2}{B} h_{i}^{\prime}(t)-\frac{b}{B} h_{i}(t) .
\end{aligned}
$$

Parameter $A$ (resp. $B$ ) represents the amplitude of postsynaptic excitatory potentials (resp. inhibitory) and $\frac{1}{a}$ (resp. $\frac{1}{b}$ ) the time constant of excitatory postsynaptic potentials (resp. inhibitory), basically representative of the kinetics of synaptic connections and delays introduced by circuitry of the dendritic tree (Freeman, 1975; Van Rotterdam et al., 1982; Jansen et al., 1993).

The function transforming the potential of the average membrane into an average pulse density by the neurons is generally assumed to be a sigmoid 
(Freeman, 1975; Dayan \& Abbott, 2001; Gerstner \& Kistler, 2002). We have followed Freeman's (1975) work for the parameterization of this function:

$$
\operatorname{sigm}(v)=\frac{2 e_{0}}{1+e^{r\left(v_{0}-v\right)}}
$$

where $2 e_{0}$ represents the maximum discharge rate, $v_{0}$ the postsynaptic potential threshold, and $r$ the sigmoid slope at $v_{0}$.

In this letter, we use the parameter values used by Jansen and Rit (1995) and Jansen et al. (1993):

$$
\left\{\begin{array}{l}
A=3.25 \mathrm{mV} \\
B=22 \mathrm{mV} \\
a=100 \mathrm{~s}^{-1} \\
b=50 \mathrm{~s}^{-1} \\
e_{0}=2.5 \mathrm{~s}^{-1} \\
v_{0}=6 \mathrm{mV} \\
r=0.56 \mathrm{mV}^{-1}
\end{array}\right.
$$

2.2 Architecture of Direct and Indirect Feedbacks. To build our model, we considered three feedback loops of the pyramidal cell population activity (see Figure 2). The first loop represents the excitatory action of pyramidal neurons on inhibitory interneurons, which in turn inhibit pyramidal activity. The second loop, which we call indirect excitation, involves the excitatory action of the main pyramidal cell population on other intermediary pyramidal cells, which in turn excite the considered population. These two loops (see Figure 2a) are present in the Jansen-Rit model. We added a third feedback loop, called direct feedback, representing the self-excitation of the pyramidal cell population. This direct feedback can replace the indirect feedback to simulate pyramidal cell excitation (see Figure 2b). Our modeling approach distinguishes between the third and second loops and differentiates between the action of interneurons directly connected to a pyramidal neuron being studied and that of the interneuron population connected to adjacent neurons. Indirect feedback is modeled using operator $\mathcal{F}_{e}$ and numbers $C_{1}$ and $C_{2}$ of synaptic connections between populations, while direct feedback is modulated by a gain parameter $G$ (see Figure $2 c$ ).

Based on the synaptic connection model described in section 2.1, we obtain the following dynamics for variables $z_{0}, z_{1}$, and $z_{2}$ that represent the outputs of the principal pyramidal cell population, the intermediary pyramidal cell population, and the interneuron population, respectively 

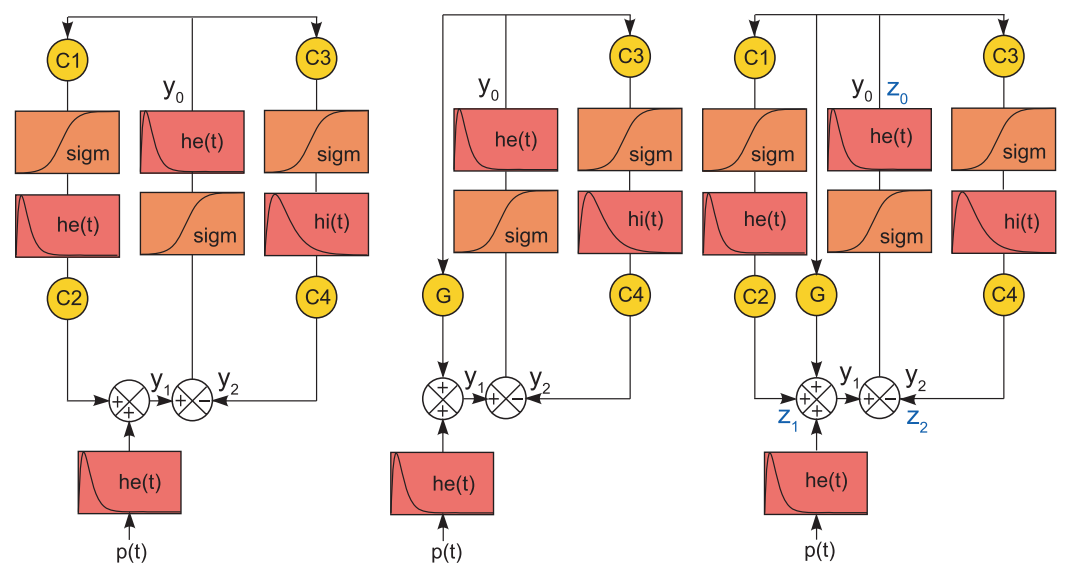

Figure 2: Neural mass model diagrams with indirect (a), direct (b), and double (c) excitatory feedbacks. Box $h_{e}(t)$ (resp. $h_{i}(t)$ ): Conversion process of action potentials into postsynaptic excitatory membrane potential (resp. inhibitor). Box sigm: Conversion process of average membrane potential into average density of action potentials discharged by neurons. Box $C_{i}$ for $i \in[1,4]$ : Coupling gain using the average number of synapses between two populations. G: Synaptic coupling gain of the direct excitatory feedback. $p(t)$ : Model excitatory input from long-distance synaptic connections with other cortical areas. Arrows: Excitatory $(+)$ and inhibitory $(-)$ actions of one population onto another.

(see Figure 2c):

$$
\begin{aligned}
& z_{0}^{\prime \prime}=A a \operatorname{sigm}\left(z_{1}+G z_{0}-z_{2}\right)-2 a z_{0}{ }^{\prime}-a^{2} z_{0}, \\
& z_{1}^{\prime \prime}=A a C_{2} \operatorname{sigm}\left(C_{1} z_{0}\right)-2 a z_{1}{ }^{\prime}-a^{2} z_{1}+A a p(t), \\
& z_{2}^{\prime \prime}=B b C_{4} \operatorname{sigm}\left(C_{3} z_{0}\right)-2 b z_{2}{ }^{\prime}-b^{2} z_{2} .
\end{aligned}
$$

As in the Jansen-Rit model, we consider the following state variables: the excitatory $\left(y_{1}=z_{1}+G z_{0}\right)$ and inhibitory $\left(y_{2}=z_{2}\right)$ inputs of the principal pyramidal cell population and the output of this population $\left(y_{0}=z_{0}\right)$. This output acts as direct excitatory feedback and on both the intermediary pyramidal cells and the inhibitory interneurons. With these variables, our model becomes

$$
\begin{aligned}
y_{0}^{\prime \prime}= & A a \operatorname{sigm}\left(y_{1}-y_{2}\right)-2 a y_{0}{ }^{\prime}-a^{2} y_{0}, \\
y_{1}^{\prime \prime}= & A a C_{2} \operatorname{sigm}\left(C_{1} y_{0}\right)+A a G \operatorname{sigm}\left(y_{1}-y_{2}\right) \\
& -2 a y_{1}^{\prime}-a^{2} y_{1}+A a p(t), \\
y_{2}^{\prime \prime}= & B b C_{4} \operatorname{sigm}\left(C_{3} y_{0}\right)-2 b y_{2}{ }^{\prime}-b^{2} y_{2} .
\end{aligned}
$$


Parameters $C_{i}, i \in[1,4]$ represent the average number of synapses between two populations. Following the formalism introduced by Jansen and Rit (1995), based on Braitenberg and Schüz (1991), we assume that each $C_{i}$ is constant and proportional to the maximum number $C$ of synapses between populations:

$$
\forall i \in[1,4], \quad C_{i}=\alpha_{i} C
$$

Parameter $G$ represents the average number of synaptic connections internal to the principal pyramidal cell population, meaning the coupling gain of direct excitatory feedback. Function $p(t)$ represents the excitatory input from non specific long-distance neuronal connections on pyramidal cells of the local model (called cortical input in Suffczynski, Kalitzin, \& Lopes da Silva, 2004).

This model generalizes both modeling approaches mentioned earlier. In fact, by stating that $G=0$, we obtain the Jansen-Rit model for which excitatory feedback is only indirect. Stating $\alpha_{2}=0$ (and therefore $C_{2}=0$ ), we obtain the model for which the excitatory feedback is only direct. To analyze the bifurcations, we write equation 2.2 as a system of first-order equations:

$$
\begin{aligned}
y_{0}^{\prime}= & y_{3}, \\
y_{1}^{\prime}= & y_{4}, \\
y_{2}^{\prime}= & y_{5}, \\
y_{3}^{\prime}= & A a \operatorname{sigm}\left(y_{1}-y_{2}\right)-2 a y_{3}-a^{2} y_{0}, \\
y_{4}^{\prime}= & A a C_{2} \operatorname{sigm}\left(C_{1} y_{0}\right)+A a G \operatorname{sigm}\left(y_{1}-y_{2}\right) \\
& -2 a y_{4}-a^{2} y_{1}+A a p(t), \\
y_{5}^{\prime}= & B b C_{4} \operatorname{sigm}\left(C_{3} y_{0}\right)-2 b y_{5}-b^{2} y_{2} .
\end{aligned}
$$

and, as main model outputs, we will consider the classical approximation of the generated LFP (Jansen et al., 1993):

$$
L F P(t)=y_{1}(t)-y_{2}(t)
$$

2.3 Parameters of Interest for Bifurcation Analysis. In section 3, we focus on system 2.3 bifurcations according to input $p(t)=p$ considered as a parameter. Choosing $p$ as a parameter of interest is a natural choice since it represents the action of the activity in other cortical units and acts as the 
excitatory input in the model. As mentioned in section 1, understanding the bifurcation diagram according to $p$, and therefore how the dynamics attractors evolve according to this parameter, allows us to know the time series features when $p(t)$ varies over time. For instance, we can deduce the different types of time series that system 2.3 can produce for $p(t)$ a gaussian variable $\mathcal{N}\left(m, \sigma^{2}\right)$ with mean $m$ and variance $\sigma^{2}$ from its bifurcation diagram for different values of parameters $C, G$, and $\alpha_{2}$. We have thereby established a glossary of identifiable behaviors in the time series underlaid by specific organizations of system 2.3 dynamics.

Parameters $G$ and $\alpha_{2}$ tune the collateral excitation physiologically existing in many brain structures (Miles \& Wong, 1986; Wang et al., 2006; Frick et al., 2008). Increase of local excitatory feedback is prompt to provoke hyperexcitability that may lead to pathological behaviors (Salin, Tseng, Hoffman, Parada, \& Prince, 1995) as epileptic discharges (McKinney, Debanne, Gähwiler, \& Thompson, 1997). In this study, we have a particular interest, in terms of generation of pathological behaviors, on the balanced effect of both excitatory feedbacks, which models a fast local feedback versus a delayed neighbor feedback.

In section 4, we use codimension 2 bifurcation diagrams (according to $p$ and $C$ ) to characterize the possible model behaviors (i.e., the types of the generated time series from those identified in section 3) for the different values of $G$ and $\alpha_{2}$. By doing so, we provide a map of the model behaviors (characterized by a type of bifurcation diagram in $(p, C)$ ) within the parameter space $\left(\alpha_{2}, G\right)$. This study allows us to analyze the model behaviors according to the weightings of direct and indirect excitatory feedbacks and their effects on the identifiable patterns in the generated time series.

\section{Bifurcation Diagrams According to $p$ and Time Series Glossary}

In this section, we describe the system bifurcation diagrams according to the input $p(t)=p$ considered as a parameter obtained for different values of parameters $C, \alpha_{2}$, and $G$. These diagrams have been built using dedicated algorithms based on efficient and well-documented numerical methods (in particular, numerical continuation) by additionally taking advantage of our knowledge of system 2.3. In the appendix we recall briefly the definitions of the bifurcations involved in this study and describe the numerical techniques developed to track them in the parameter space.

The bifurcation diagram enables us to determine the interesting intervals of $p$ values and trace the evolution of geometric invariants (e.g., singular points, limit cycles, homoclinic connections) that organize the dynamics. For each organization, the model generates specific types of time series for input $p(t)$ varying over time and from which we can derive qualitative properties using the bifurcation diagram. We associate a name, an acronym, and a colored flag with each type of time series, relying on its fundamental properties. For each case, we provide a figure with, on the left, the 


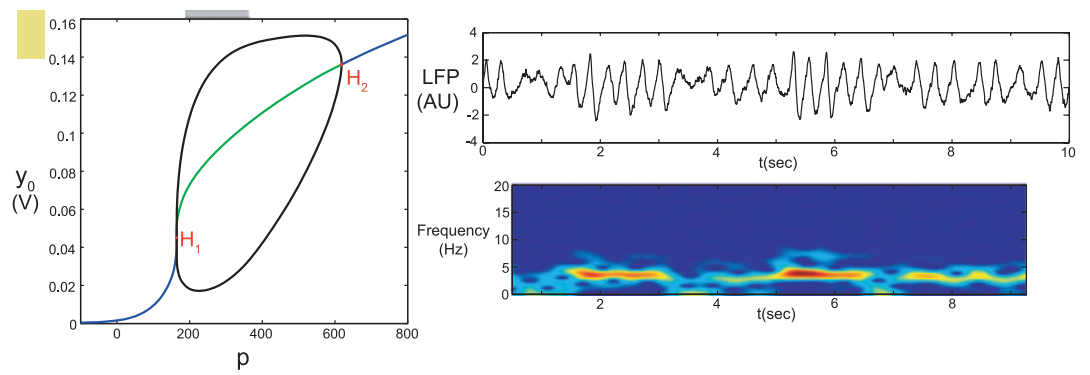

Figure 3: Bifurcation diagram (left) according to $p$ and instance of LFP time series and its spectrogram (right) associated with the NMO case $\left(G=25, \alpha_{2}=\right.$ 0.3 , and $C=130$ for the simulation). Blue curves: Stable singular points. Green curve: Singular points with four associated eigenvalues with negative real parts. Red points: Supercritical Hopf bifurcations $\left(\mathrm{H}_{1}\right.$ and $\left.\mathrm{H}_{2}\right)$. Black curves: Maximal and minimal $y_{0}$ values along the stable cycles. Horizontal gray bar: Confidence interval $[\langle p\rangle-\sigma,\langle p\rangle+\sigma]$ of the gaussian variable $p(t)$ used to generate the time series.

bifurcation diagram and the dynamics organizations ( $y_{0}$ traces of the singular points and minimal and maximal $y_{0}$ values along each limit cycle) and, on the right, an instance of associated LFP time series obtained with $p(t)$ a gaussian variable and its time-frequency diagram.

We have focused on the time series specifically characterized by their qualitative properties, in particular, the types of oscillations. We are not presenting all of the possible bifurcation diagrams because different diagrams can generate the same type of time series as long as they share essential structural properties. We mention the slightly different cases in the text. We do not discuss trivial cases where there is no limit cycle. These cases, where the only attractors are fixed points, correspond either to the activity of a silent population or a nonphysiological activity where the LFP remains high for a long period of time. For the time series generation, the mean and variance of the gaussian variable $p(t)$ are selected so that $p(t)$ takes its values in the interval of interest with an expectancy greater than 0.5 .

3.1 Noise Modulated Oscillations (NMO)_Figure 3. For each value of $p$, system 2.3 admits a unique singular point that is stable (blue) if $p<p_{\mathrm{H}_{1}}$ or $p>p_{\mathrm{H}_{2}}$ and unstable (green) if $p_{\mathrm{H}_{1}}<p<p_{\mathrm{H}_{2}}$ (see Figure 3). At $p=p_{\mathrm{H}_{1}}$, the system undergoes a supercritical Hopf bifurcation giving birth to a stable limit cycle that persists for $p>p_{\mathrm{H}_{1}}$ and disappears for $p=p_{\mathrm{H}_{2}}$ through another supercritical Hopf bifurcation. In the presence of an input varying over time (especially for a gaussian variable), the generated time series 


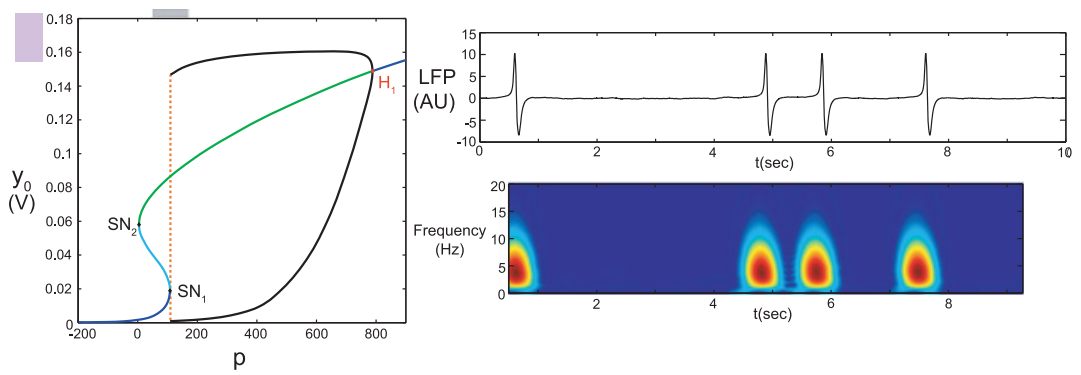

Figure 4: Bifurcation diagram (left) according to $p$ and instance of LFP time series and its spectrogram (right) associated with the NIS case $\left(G=60, \alpha_{2}=0.5\right.$, and $C=150$ for the simulation). Blue curves: Stable singular points. Cyan (resp. green) curve: Singular points with five (resp. four) associated eigenvalues with negative real parts. Black points: Saddle node bifurcations $\mathrm{SN}_{1}$ and $\mathrm{SN}_{2}$. Black curves: Maximal and minimal $y_{0}$ values along the stable cycles. Dashed orange line: SNIC Bifurcation (saddle node on invariant cycle). Horizontal gray bar: Confidence interval $[\langle p\rangle-\sigma,\langle p\rangle+\sigma]$ of the gaussian variable $p(t)$ used to generate the time series.

oscillates when $p_{\mathrm{H}_{1}}<p(t)<p_{\mathrm{H}_{2}}$ and oscillation amplitude and frequency are modulated by the input value.

In a slightly different case (in particular, for a greater $C$ value), the Hopf bifurcation $\mathrm{H}_{1}$ is subcritical. A fold bifurcation of limit cycles occurs for a value $p_{\mathrm{FLC}}<p_{\mathrm{H}_{1}}$ at which stable and unstable limit cycles collide and disappear. Unstable and stable cycle families indexed by $p$ connect $\mathrm{H}_{1}$ and $\mathrm{H}_{2}$, respectively. Since the most important property to the existence of stable cycle families is preserved, the time series generated in this case are the same type as those shown in Figure 3.

3.2 Noise Induced Spiking (NIS)_Figure 4. The curve of singular points is S-shaped and points $\mathrm{SN}_{1}$ and $\mathrm{SN}_{2}$ corresponding to saddle node bifurcations split it into three branches (see Figure 4). We refer to the subsets of singular points verifying $y_{0}<y_{\mathrm{SN}_{1}}, y_{0}>y_{\mathrm{SN}_{2}}$, and $\left.y_{0} \in\right] y_{\mathrm{SN}_{1}}, y_{\mathrm{SN}_{2}}$, respectively, as low branch, high branch, and middle branch. The points on the low branch are stable (blue), and those on the middle branch are unstable (cyan). The points on the high branch are unstable (green) for $p<p_{\mathrm{H}_{1}}$ and stable (blue) alternatively. We describe the diagram for the decreasing $p$ values. For $p>p_{\mathrm{H}_{1}}$ the system admits a unique singular point that is stable. At $p=p_{\mathrm{H}_{1}}$, a supercritical Hopf bifurcation $\mathrm{H}_{1}$ creates a stable limit cycle that persists for small $p$ values until it disappears through a SNIC bifurcation (saddle node on invariant cycle, sometimes referred to as saddle 


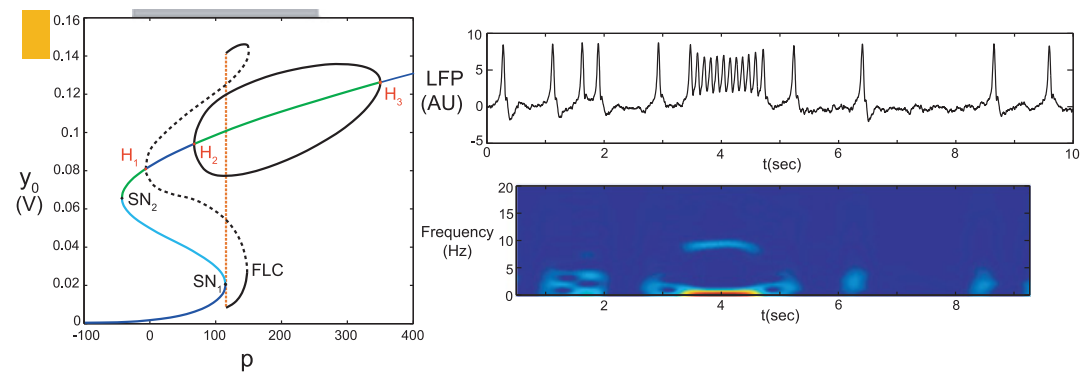

Figure 5: Bifurcation diagram (left) according to $p$ and instance of LFP time series and its spectrogram (right) associated with the NIS-OTO case $\left(G=0, \alpha_{2}=\right.$ 0.8 , and $C=136$ for the simulation). Blue curves: Stable singular points. Cyan (resp. green) curve: Singular points with five (resp. four) associated eigenvalues with negative real parts. Red points: Hopf bifurcations $\left(\mathrm{H}_{1}\right.$ subcritical, $\mathrm{H}_{2}$ and $\mathrm{H}_{3}$ supercritical). Black points: Saddle node bifurcations $\mathrm{SN}_{1}$ and $\mathrm{SN}_{2}$. Black curves: Maximal and minimal stable cycle $y_{0}$ values along the stable cycles. Dashed black curve: Maximal and minimal $y_{0}$ values along the stable cycles. Dashed orange line: SNIC bifurcation (saddle node on invariant cycle). Horizontal gray bar: Confidence interval $[\langle p\rangle-\sigma,\langle p\rangle+\sigma]$ of the gaussian variable $p(t)$ used to generate the time series.

node homoclinic bifurcation) at $p=p_{\mathrm{SN}_{1}}$. For $p<p_{\mathrm{SN}_{1}}$ the system admits a unique stable singular point.

For input $p(t)$ with an average chosen close to $p_{\mathrm{SN}_{1}}$, the system alternates between a stable singular point on the low branch and the high-amplitude limit cycle. The period of the cycle is quite large since it is close to the SNIC bifurcation. Hence, the generated time series display alternations of spikes and long quiescence phases. We call this mechanism noise-induced spiking (NIS).

\subsection{Noise Induced Spiking and Over Threshold Oscillations (NIS-} OTO)-Figure 5. The curve of singular points is split into three branches (low, middle, and high) by two saddle node bifurcation points $\mathrm{SN}_{1}$ and $\mathrm{SN}_{2}$ as in the NIS case.

The singular points of the low branch are stable (blue), and those of the middle branch are unstable (cyan) (see Figure 5). The points of the high branch are unstable (green) for $p \in] p_{\mathrm{SN}_{2}}, p_{\mathrm{H}_{1}}[\cup] p_{\mathrm{H}_{2}}, p_{\mathrm{H}_{3}}$ [ and stable alternatively. The system undergoes a subcritical Hopf bifurcation at $p=$ $p_{\mathrm{H}_{1}}$ and two supercritical Hopf bifurcations at $p=p_{\mathrm{H}_{2}}$ and $p=p_{\mathrm{H}_{3}}$. For $p<p_{\mathrm{H}_{1}}$ and $p>p_{\mathrm{H}_{3}}$, the system admits a stable point as a single attractor. At $p=p_{\mathrm{H}_{1}}$, the subcritical Hopf bifurcation gives birth to an unstable limit cycle that persists for greater values of $p$. At $p=p_{\text {FLC }}$, it disappears through 


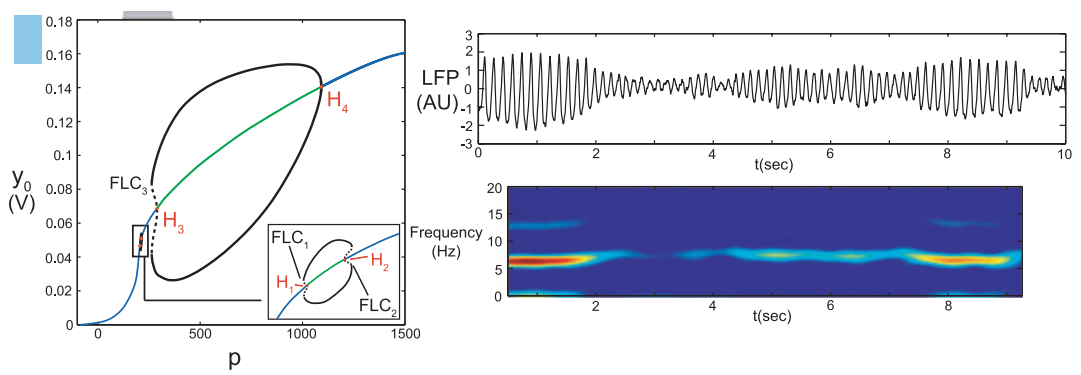

Figure 6: Bifurcation diagram (left) according to $p$ and instance of LFP time series and its spectrogram (right) associated with the NITAM case ( $G=0$, $\alpha_{2}=0.3$, and $C=151$ for the simulation). Blue curves: Stable singular points. Green curves: Singular points with four associated eigenvalues specific to the negative real part. Red points: Hopf bifurcations $\left(\mathrm{H}_{1}, \mathrm{H}_{2}\right.$, and $\mathrm{H}_{3}$ subcritical and $\mathrm{H}_{4}$ supercritical). Black curves: Maximal and minimal $y_{0}$ values along the stable cycles. Dashed black curves: Maximal and minimal $y_{0}$ values along the stable cycles. Horizontal gray bar: Confidence interval $[\langle p\rangle-\sigma,\langle p\rangle+\sigma]$ of the gaussian variable $p(t)$ used to generate the time series.

a fold bifurcation of limit cycles with a high-amplitude stable limit cycle. This latter stable cycle exists for $p$ between $p_{\mathrm{SN}_{1}}$, corresponding to a SNIC bifurcation, and $p_{\text {FLC }}$. Moreover, a family of low-amplitude stable limit cycles connects $\mathrm{H}_{2}$ and $\mathrm{H}_{3}$.

When considering a time-varying input $p(t)$, the generated time series alternates high-amplitude oscillations (for $p(t) \in] p_{\mathrm{H}_{1}}, p_{\mathrm{FLC}}[$ ), low-amplitude oscillations for $p(t) \in] p_{\mathrm{H}_{2}}, p_{\mathrm{H}_{3}}$ [ and quiescence phases. We call this type of time series noise-induced spiking and over-threshold oscillations (NIS-OTO).

\subsection{Noise Induced Thresholded Amplitude Modulation (NITAM) -}

Figure 6. For each value of $p$, the system admits a unique singular point (see Figure 6). This point is unstable (green) for $p \in] p_{\mathrm{H}_{1}}, p_{\mathrm{H}_{2}}[\cup] p_{\mathrm{H}_{3}}, p_{\mathrm{H}_{4}}$ [ and stable (blue) alternatively. For $\left.p<p_{\mathrm{FLC}_{1}}, p \in\right] p_{\mathrm{FLC}_{2}}, p_{\mathrm{FLC}_{3}}\left[\right.$, and $p>p_{\mathrm{H}_{4}}$ the system admits a stable point as a unique attractor. When $p=p_{\mathrm{H}_{1}}$, the system undergoes a subcritical Hopf bifurcation that gives birth to a family of unstable cycles that disappear when $p=p_{\mathrm{FLC}_{1}}$ by a fold bifurcation of limit cycles with a stable cycle. This stable cycle appears and disappears through fold bifurcation of limit cycles at $p=p_{\mathrm{FLC}_{1}}$ and $p=p_{\mathrm{FLC}_{2}}$, respectively. At $p=p_{\mathrm{FLC}_{2}}$, the stable cycle collides with the unstable cycle that disappears at $p=p_{\mathrm{H}_{2}}$ through subcritical Hopf bifurcation. When $p=p_{\mathrm{H}_{3}}$, an unstable limit cycle emerges from a subcritical Hopf bifurcation and disappears by 


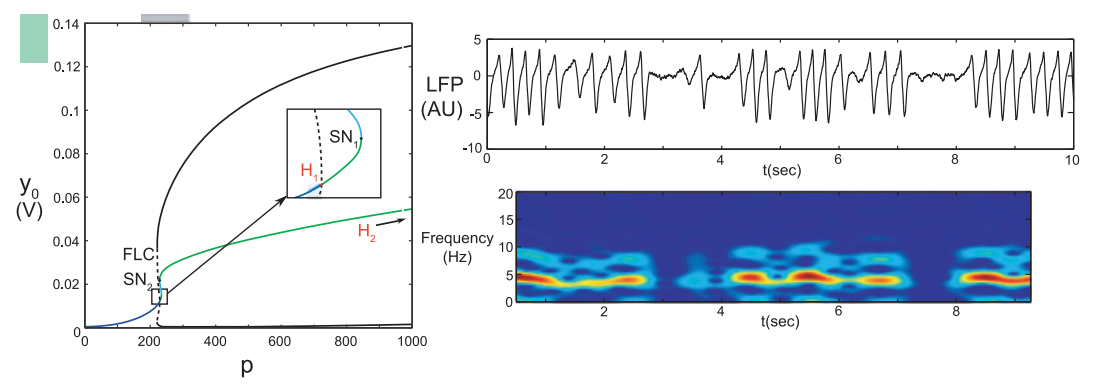

Figure 7: Bifurcation diagram (left) according to $p$ and instance of LFP time series and its spectrogram (right) associated with the NIS-STO case $(G=0$, $\alpha_{2}=0.3$ and $C=300$ for the simulation). Blue curve: Stable singular points. Cyan (resp. green) curve: Singular points with five (resp. four) associated eigenvalues with negative real parts. Red points: Hopf bifurcation $\left(\mathrm{H}_{1}\right.$ subcritical and $\mathrm{H}_{2}$ supercritical). Black points: Saddle node bifurcation $\left(\mathrm{SN}_{1}\right.$ and $\left.\mathrm{SN}_{2}\right)$. Black curves: Maximal and minimal $y_{0}$ values along the stable cycles. Dashed black curve: Maximal and minimal $y_{0}$ values along the unstable cycles. Horizontal gray bar: Confidence interval $[\langle p\rangle-\sigma,\langle p\rangle+\sigma]$ of the gaussian variable $p(t)$ used to generate the time series.

a fold bifurcation of limit cycles at $p=p_{\mathrm{FLC}_{3}}$ with a stable limit cycle that persists until $p=p_{\mathrm{H}_{4}}$ where it disappears by supercritical Hopf bifurcation.

By choosing a noise $p(t)$ with an average close to the transition between both stable cycles, the generated time series alternates between lowand high-amplitude oscillations due to the presence of stable limit cycles for $p_{\mathrm{FLC}_{1}}<p(t)<p_{\mathrm{FLC}_{2}}$, and $p_{\mathrm{FLC}_{3}}<p(t)<p_{\mathrm{H}_{4}}$, respectively. Quiescence phases can also appear when the point along the orbit follows the stable point of the low branch. We call this type of behavior noise-induced thresholded amplitude modulation (NITAM).

\subsection{Noise Induced Spiking and Subthreshold Oscillations (NIS-} STO)-Figure 7. The set of singular points is split into three branches (low, middle, and high) by two saddle node bifurcations $\mathrm{SN}_{1}$ and $\mathrm{SN}_{2}$ as in the NIS case (see Figure 7). The singular points of the middle branch are unstable (cyan). Those of the low branch are stable (blue) for $p<p_{\mathrm{H}_{1}}$ and unstable (green) alternatively. Furthermore, the points of the high branch are unstable (green) for $p<p_{\mathrm{H}_{2}}$ and stable (blue) alternatively. At $p=p_{\mathrm{H}_{1}}$, the system undergoes a subcritical Hopf bifurcation, creating an unstable cycle that persists for smaller values of $p$ until it disappears at $p=p_{\text {FLC }}$ through a fold bifurcation of limit cycles with a stable cycle. This latter stable cycle exists for $p$ greater than $p_{\mathrm{FLC}}$ and disappears at $p=p_{\mathrm{H}_{2}}$ (not shown in Figure 7) through a supercritical Hopf bifurcation. 
For a gaussian input $p(t)$ with an average close to $p_{\mathrm{H}_{1}}$, the time series displays an alternation of large oscillations, quiescence phases reflecting the input noise, and subthreshold oscillations. The large oscillations result from the presence of the stable cycle for $p(t)>p_{\text {FLC }}$. The quiescence phases correspond to periods of time during which the current point is close to the low branch of stable point $\left(p(t)<p_{\mathrm{H}_{1}}\right)$. The subthreshold oscillations occur in the transitions between the two preceding regimes and result from the repulsiveness of the singular point on the low branch when $p(t) \in] p_{\mathrm{FLC}}, p_{\mathrm{H}_{1}}[$. We call this type of behavior noise-induced spiking and subthreshold oscillations (NIS-STO).

A similar dynamical organization was found by Liley and Walsch (2013) in a mean field model designed to reproduce the burst suppression during anesthesia. In this context, the Hopf bifurcation is supercritical, and a family of small, stable limit cycles emerges connecting the family of unstable limit cycles at an additional fold bifurcation of limit cycles. In a certain region of the parameter space, this model is able to reproduce small oscillations between high-amplitude bursts. Burst suppression emerges in the Liley model by the addition of a slow system driven by the mean fields. Therefore, several physiologically plausible hypotheses are given by the authors to explain the genesis of EEG bursting. One of them concerns slow changes of GABA and glutamate neuromodulations in activity. However, neuromodulations in activity may not be sufficient to account for burst suppression, and other mechanisms need to be considered.

\section{Impact of the Balance between Direct and Indirect Feedbacks}

Direct and indirect feedbacks have an essential impact on the activity of the neuronal population being studied, which results in different oscillation profiles in the model outputs. Note that indirect feedback means intermediary pyramidal cells, and therefore its coupling gain is defined in the model as a proportion $\alpha_{2} \in[0,1]$ of the maximum number $C$ of synaptic connections between populations. An analysis of the relative effects of direct and indirect feedbacks requires taking into account parameter $C$ modulating all the synaptic interactions between populations in addition to parameters $\alpha_{2}$ and $G$ (coupling gain of direct feedback). Specifically for fixed values of $\alpha_{2}$ and $G$, the model can generate different types of time series among those described in section 3 depending on the value of $C$. We can identify them using the codimension 2 bifurcation diagrams according to parameters $C$ and $p$. When we modify values $\alpha_{2}$ and $G$, this diagram changes and therefore so does the time series panel that system 2.3 can generate.

In this section, we describe the codimension 2 bifurcation diagrams of system 2.3 according to $C$ and $p$ and their distribution in the rectangle $(G$, $\left.\alpha_{2}\right) \in[0,80] \times[0,1]$. We obtain a partition of this rectangle and, for each region representing a scale of direct and indirect feedback gains, a panel of 


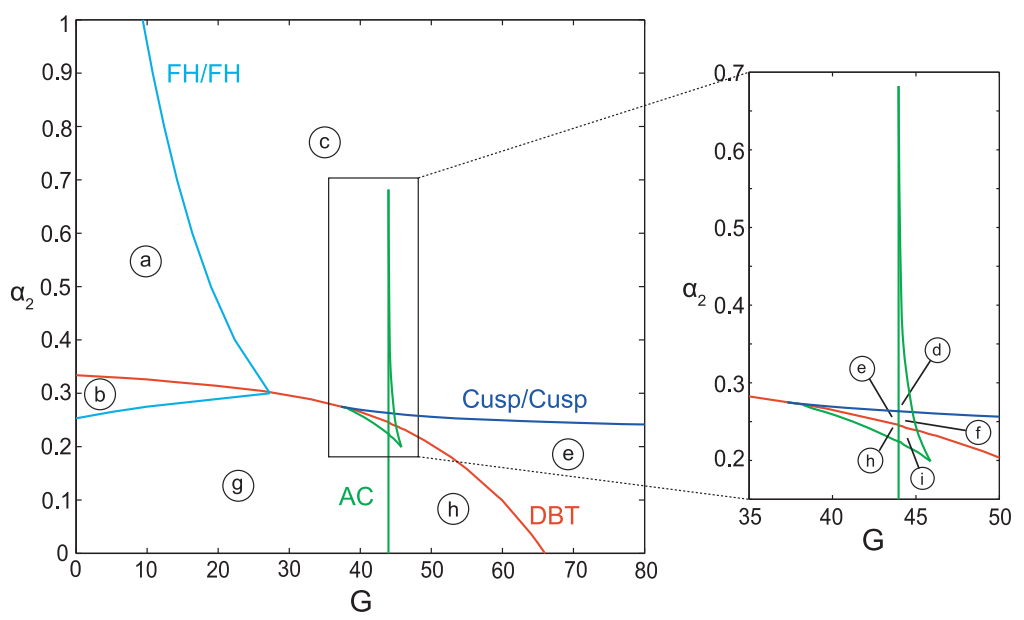

Figure 8: Partition of the rectangle $[0,80] \times[0,1]$ of $\left(G, \alpha_{2}\right)$ values according to the type of bifurcation diagram in $(C, p)$. The picture on the right is a zoom on the center part of the rectangle. Cyan curve: Appearance or disappearance of two folds of the Hopf branch. Red curve: Degenerated Bogdanov-Takens bifurcation. Blue curve: Cusp-cusp bifurcation. Green curve: Appearance or disappearance of at least one cusp (Cusp or Cusp $_{1} / \mathrm{Cusp}_{2}$ couple). These curves define a partition of the rectangle into 11 regions indexed from a to $\mathrm{i}$; each region is characterized by a type of codimension 2 bifurcation diagram $(C, p)$ represented by the associated panel in Figure 9 or 10.

possible behaviors for system 2.3. In the appendix, we describe briefly the codimension 2 bifurcations and the dedicated numerical methods used to locate them in $(C, p)$ plane and to build the partition of $\left(G, \alpha_{2}\right)$ plane.

\subsection{Codimension 2 Bifurcation Diagram in $(C, p)$ and Partition in} $\left(\alpha_{2}, G\right)$. First, it should be noted that the interval [0,80] of $G$ values is consistent with the values studied in the literature (Huneau et al., 2013). In addition, rectangle $[0,80] \times[0,1]$ contains the essential codimension 3 and 4 bifurcations undergone by system 2.3 based on parameters $p, C, G$, and $\alpha_{2}$. There are other behaviors of less interest for greater $G$ values considered to be far from the physiology. For clarity when presenting the behaviors, we have chosen not to describe these behaviors beyond the limits of the model.

Figure 8 shows the partition of rectangle $[0,80] \times[0,1]$ values of $\left(G, \alpha_{2}\right)$. Each region from (a) to (i) is related to a type of codimension 2 diagram according to $(C, p)$ shown in Figure 9 (for cases (a) to (f)) or Figure 10 (for cases (g), (h), and (i)). In each bifurcation diagram, we have highlighted the intervals of $C$ values for which the model generates a given type of outputs from those presented in section 3. Each interval is materialized by a colored 

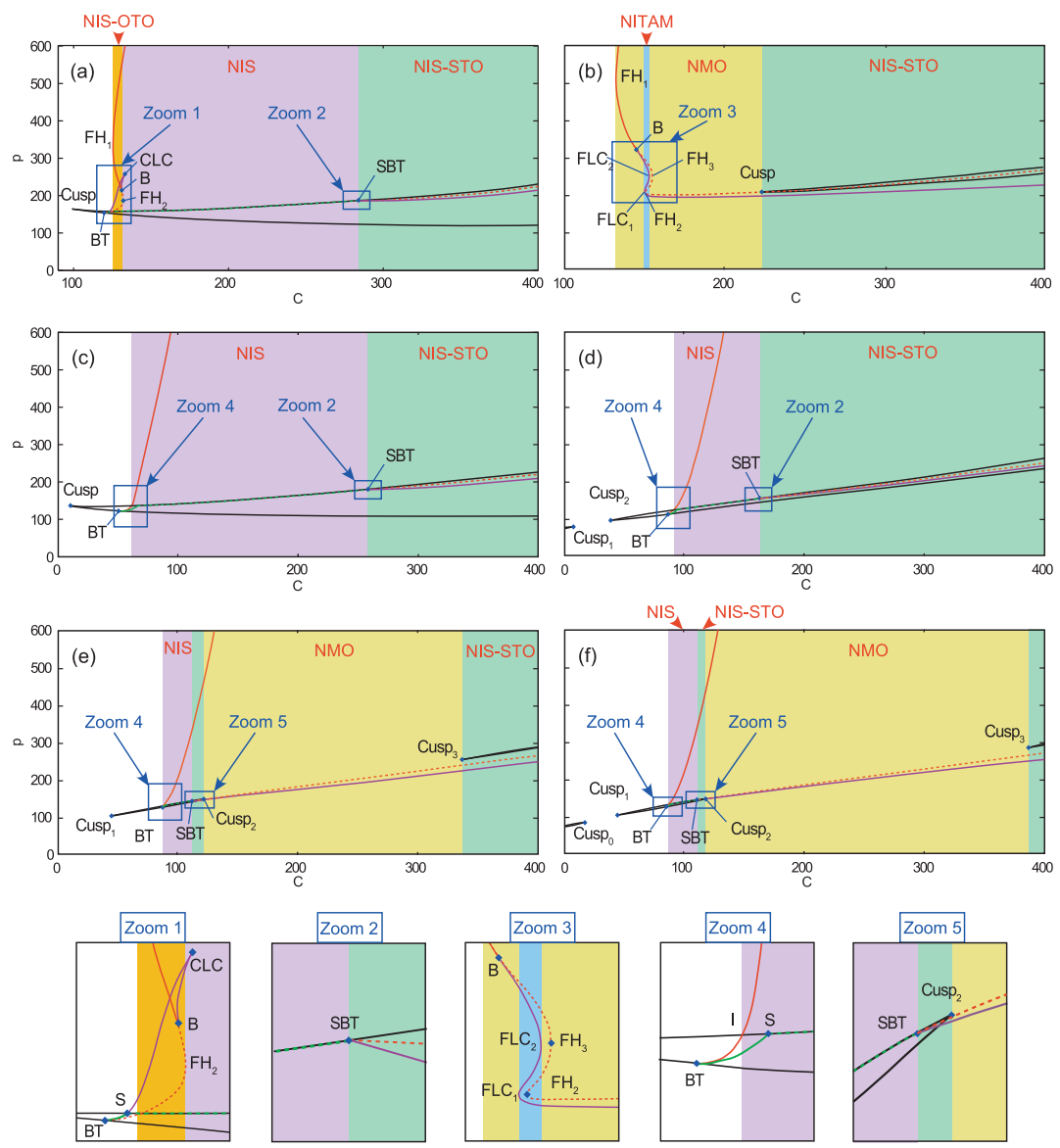

Figure 9: Bifurcation diagrams according to parameters $C$ and $p$ in each region (a) $-(\mathrm{f})$ of the partition of the rectangle $[0,80] \times[0,1]$ of $\left(G, \alpha_{2}\right)$ values shown in Figure 8. The five lower panels are zoom shots of the parts of diagrams (a) to (f) indicated with a blue rectangle and labeled with Zoom1 to Zoom5. Codimension 1 bifurcations (curves): saddle node in black, supercritical Hopf in a solid red line, subcritical Hopf in a dotted red line, homoclinic connection in green, SNIC in green dots, fold of limit cycles in purple. Codimension 2 bifurcations (blue diamonds): Cusp, Bogdanov-Takens (BT), Bogdanov-Takens with SNIC (SBT), Bautin (B), homoclinic connection to SNIC (S), cusp of limit cycles (CLC). In each diagram, the intervals of $C$ values corresponding to a given type among those described in section 3 are identified by colored bands: NMO in yellow, NIS in mauve, NIS-OTO in orange, NITAM in blue, NIS-STO in green. The intervals of $C$ values left blank correspond to trivial cases. 

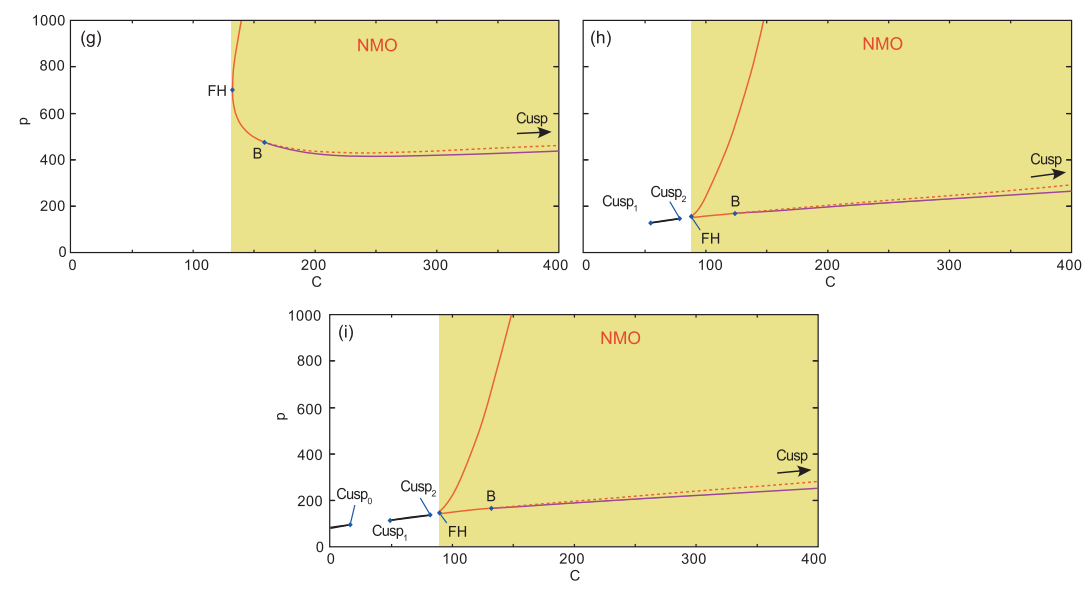

Figure 10: Bifurcation diagrams according to parameters $C$ and $p$ in each region (g)-(i) of the partition of the rectangle $[0,80] \times[0,1]$ of $\left(G, \alpha_{2}\right)$ values shown in Figure 8. Codimension 1 bifurcations (curves): Saddle node in black, supercritical Hopf in a solid red line, subcritical Hopf in a dotted red line, fold of limit cycles in purple. Codimension 2 bifurcations (blue diamonds): Cusp and Bautin (B). In each diagram, the intervals with a value of $C$ corresponding to the NMO type are identified by the yellow rectangles. The intervals of $C$ values left blank correspond to trivial cases. In these three cases, there exists a cusp bifurcation for a high value of $C$ (greater than 400).

vertical band corresponding to the type of related outputs (see the flags to the left of Figures 3 to 7 ).

In all the diagrams $(C, p)$ shown in Figures 9 and 10, the following codimension 1 bifurcations occur along curves (or branches):

- Supercritical Hopf: Solid red lines

- Subcritical Hopf: Dotted red lines

- Saddle node: Black lines

- Homoclinic connection: Solid green lines

- SNIC : Dotted green lines

- Folds of limit cycles: Purple lines

The codimension 2 bifurcations occur at the points indicated by a blue diamond and the following abbreviations according to their nature:

- Cusp,

- Bogdanov-Takens (BT)

- Bautin (B)

- Bogdanov-Takens in the presence of a SNIC (SBT)

- Cusp of limit cycles (CLC) 
These codimension 2 bifurcations split the interval $[0,400]$ of $C$ values into intervals corresponding to a type of bifurcation diagrams according to $p$ among those presented in section 3. Other points play a similar role and are mentioned in Figures 9 and 10:

- Fold point of a branch of fold bifurcations of limit cycle (FLC)

- Fold point of a Hopf bifurcation branch (FH)

- Intersection of a Hopf bifurcation branch with a saddle node bifurcation branch (I)

Note that in order to prevent confusion, the FH and I type points should be distinguished from the Hopf-Hopf (also called double-Hopf) bifurcation and the fold-Hopf (also called zero-Hopf) bifurcation. In fact, an FH point is not a transverse intersection of two Hopf bifurcation branches but simply a fold that, for increasing values of $C$, gives birth to two Hopf bifurcations for distinct $p$ values. Similarly, at point $I$, the singular point undergoing the Hopf bifurcation is not one of the singular points disappearing through the saddle node bifurcation. The intersection of the two branches simply separates the cases for which limit cycles exist from those that do not, around a singular point for the value of $p$ corresponding to the saddle node bifurcation of two other singular points. In the following, for a point $Q$ in a bifurcation diagram in $(C, p)$, we call $C_{Q}$ and $p_{Q}$ its $C$ and $p$ components, respectively: $Q=\left(C_{Q}, p_{Q}\right)$.

In each type of diagram, from (a) to (i), there is an interval of $C$ values (blank in the panels shown in Figures 9 and 10), bounded by 0 on the left, that corresponds to three trivial cases. In the first case, for every $p$ value, there is a single singular point for system 2.3 that is attractive. This is the case, for example, for $C \in\left[0, C_{\text {Cusp }}\right]$ in the type (a) diagram or for $C \in\left[0, C_{\mathrm{FH}_{1}}\right]$ in the type (b) diagram. Therefore, the time series produced with $p(t)$, a gaussian input, directly reflects the input noise. The second trivial case appears when a cusp bifurcation occurs for $C=C_{\text {Cusp }}$, creating two branches of saddle node bifurcations according to $p$ (called inferior and superior branches in reference to the $p$ values). For a certain interval of $p$ values, system 2.3 is therefore bistable: two stable singular points coexist. This case takes place, for example, for $C \in\left[C_{\text {Cusp }}, C_{\mathrm{BT}}\right]$ in (a) and (c) type diagrams and for $C \in\left[C_{\mathrm{Cusp}_{1}}, C_{\mathrm{BT}}\right]$ in (d) to (f) type diagrams. The time series produced with $p(t)$, a gaussian input, displays hysteresis behavior between two values of potential (low and high), and the period of time surrounding the high potential is too long to represent a physiological output. The system can then undergo a Bogdanov-Takens bifurcation for $C=C_{\mathrm{BT}}$, as in diagrams (a) and (c) to (f), producing on the lower branch of saddle node bifurcations. This codimension 2 bifurcation therefore gives birth to a Hopf bifurcation branch (in red). For $C$ fixed close to $C_{\mathrm{BT}}$, the limit cycle born from the Hopf bifurcation persists for close values of $p$ but quickly disappears by homoclinic connection with a saddle. Therefore, a branch of homoclinic 
connections (in green) also starts from the Bogdanov-Takens bifurcation point. For $C$ close to $C_{\mathrm{BT}}$, the cycle born from Hopf bifurcation is not large enough to break the bistability between two stable points, and the generated time series are, once again, nonphysiological. The cases identified in section 3 appear for $C$ values that are greater than a bifurcation value whose nature varies depending on diagrams (a) to (i). In the following, we present the regions in increasing order of complexity. Cases $C \in\left[\mathrm{C}_{\mathrm{FH}_{1}}, C_{\mathrm{FH}_{2}}\right]$ in (a) (red rectangle) and $C \in\left[C_{\mathrm{FH}_{1}}, C_{\mathrm{FH}_{2}}\right]$ in (b) (blue rectangle), are described at the end of this section.

Regarding diagrams (b), (g), (h), and (i), two supercritical Hopf bifurcation branches appear for $C=C_{\mathrm{FH} 1}$. For a value of $C>C_{\mathrm{FH} 1}$ fixed in the interval shown in yellow, a family of limit cycles indexed by $p$ connect the two bifurcations. This case corresponds to the diagram in Figure 3 and produces time series of NMO type.

Regarding diagrams (c) to (f), the branch of Hopf bifurcations resulting from the Bogdanov-Takens BT bifurcation intersects the upper branch of the saddle node at $C=C_{\mathrm{I}}$ (see zoom 4 ). This transition changes the trivial bistability case between two stable singular points into bistability between a stable singular point and a stable cycle. In addition, the branch of homoclinic connections resulting from the BT becomes a branch of SNIC bifurcations (green dotted line) starting with a $C$ value close to $C_{I}$, that is, when it meets the upper branch of the saddle node. For an interval of $C$ values shown with a mauve rectangle on the panels in Figure 9, the system undergoes, for decreasing $p$ values, a supercritical Hopf bifurcation creating a limit cycle that disappears through a SNIC bifurcation (homoclinic connection on a saddle node) and, finally, a second saddle node. This type of diagram corresponds to the case in figure 4 for which the generated time series are of the NIS type.

When $C$ is increased again in these same diagrams (c) to ( $\mathrm{f}$ ), as well as diagram (a), a Bogdanov-Takens bifurcation in the presence of a SNIC (SBT) occurs for $C=C_{\mathrm{SBT}}$ on the upper branch of the saddle node bifurcations (see zoom 2). The SNIC branch is interrupted at this point to become a single saddle node for $C$ values greater than $C_{\mathrm{SBT}}$. The cycle born from the Hopf bifurcation (for a very high $p$ value) then persists down to a $p$ value that is less than that of the saddle node branch. A subcritical Hopf bifurcation branch creates a family of unstable cycles also created at $C=C_{\mathrm{BT}}$ as well as a branch of fold bifurcations of limit cycles corresponding to $p$ values for which the stable and unstable cycles collide and disappear. For intervals of $C$ values shown in green in the panels of Figure 9, the bifurcation diagram according to $p$ is therefore similar to the diagram in Figure 7 and the time series generated for a gaussian input $p(t)$ are of NIS-STO type.

For diagrams (e) and (f), the two branches of saddle node bifurcations collide and disappear via a cusp bifurcation at $C=C_{\mathrm{Cusp}_{2}}$ (see zoom 5), only to reappear at $C=C_{\mathrm{Cusp}_{3}}$. For a fixed value of $C$ in $\left[C_{\mathrm{Cusp}_{2}}, C_{\mathrm{Cusp}_{3}}\right]$, 
a family of limit cycles indexed by $p$ connects both supercritical (upper branch) and subcritical (lower branch) Hopf bifurcations. The bifurcation diagram according to $p$ is therefore similar to that of Figure 3, and the outputs generated are of NMO type. The $\mathrm{Cusp}_{3}$ bifurcations for (e) and (f) (resp. Cusp for (b)) make the system bistable again for $C>C_{\mathrm{Cusp}_{3}}$ (resp. $C>C_{\text {Cusp }}$ for (b)) and transform the NMO type diagram into a NIS-STO type (Figure 7).

We conclude with the description of the cases where, for the same $C$ value, more than two Hopf bifurcations appear in the bifurcation diagram according to $p$ : NIS-OTO types (orange) for a and NITAM (blue) for (b). In (a), the Hopf bifurcation curve emanating from the Bogdanov-Takens bifurcation admits two folds defining an interval $] C_{\mathrm{FH}_{1}}, C_{\mathrm{FH}_{2}}$ [ of $C$ values (orange rectangle) for which there are three Hopf bifurcations with different $p$ values. This case presents a complex series of bifurcation diagrams according to $p$ (see zoom 1). However, all of these diagrams share the essential features of that of Figure 5 from the time series generation viewpoint (see Touboul et al., 2011, for illustrations of all the bifurcation diagrams according to $p$ in this case). Hence, for $C$ in the orange rectangle, the model generates time series of NIS-OTO type. The highest Hopf bifurcation (i.e., the greatest $p$ value) gives birth to a branch of limit cycles, which either connects to the middle Hopf bifurcation (diagram in Figure 5) or to the SNIC via two fold bifurcations of limit cycles. In either situation, two small-amplitude limit cycles coexist respectively for $p$ values close to that corresponding to the saddle node bifurcation. As $p$ increases, the large cycle disappears, whereas the small cycle persists. This persistence leads to NIS-OTO-type time series for a gaussian input $p(t)$.

Finally, in diagram (b), the Hopf bifurcation branch shows two other folds in $C=C_{\mathrm{FH}_{2}}$ and $C=C_{\mathrm{FH}_{3}}$ in addition to the one at $C=C_{\mathrm{FH}_{1}}$, and a Bautin bifurcation branch turns the supercritical Hopf bifurcation into a subcritical one. Hence, for each fixed value of $C \in] C_{\mathrm{FH}_{1}}, C_{\mathrm{FH}_{2}}$ [ (resp. $C \in$ $\mathrm{C}_{\mathrm{FH}_{2}}, C_{\mathrm{FH}_{3}}$ [) (blue rectangle), the system undergoes two (resp. four) Hopf bifurcations when $p$ varies. The bifurcations for the two smallest $p$ values are connected by a family of limit cycles with an amplitude that remains small, whereas the two bifurcations for the two greatest $p$ values are connected by a family of limit cycles with an amplitude that becomes large. At the Bautin bifurcation, a branch of fold bifurcations of limit cycles emerges corresponding to a fold in the family of limit cycles connecting the two upper Hopf bifurcations. In both cases, these families can undergo the fold bifurcation of limit cycles transforming the stable cycle into an unstable one, as shown in Figure 6. Just as in diagram (a), we do not differentiate between the cases where these folds exist organized by the Bautin bifurcation and the cases where they do not exist, since the types of the time series are similar in both cases. They alternate between periods of small and large amplitude and are both of NITAM type (see Figure 6). 
4.2 Transitions between Regions. The partition of the rectangle $[0,80] \times[0,1]$ in Figure 8 identifies the regions in which the codimension 2 bifurcation diagrams according to $(C, p)$ are diffeomorphic. Neighboring regions of this partition are separated by curves, some of them corresponding to bifurcations of codimension 3 or greater. Others separate the cases where a codimension 1 bifurcation branch (in particular, the Hopf bifurcation branch) displays or does not display a fold for a certain value of $C$. In this section, we describe these transitions between regions by relying on the comparison between pairs of diagrams among (a) to (i) in Figures 9 and 10.

The red curve (separating (a) and (b), (c) and (g), (e) and (h), and (f) and (i)) is a degenerate Bogdanov-Takens bifurcation (Dumortier, Roussarie, Sotomayor, \& Zoladek, 1991; Baer, Kooi, Kuznetsov, \& Thieme, 2006) occurring with a cusp bifurcation. Roughly speaking, this codimension 3 bifurcation corresponds to the split of a continuous Hopf bifurcation branch into two branches by a cusp bifurcation. For system 2.3, from the perspective of $(C, p)$, it involves the appearance of the two Bogdanov-Takens bifurcations (diagrams (a), (c), (e), and (f)): BT occurs on the lower saddle node branch (lowest $p$ values), which results, in particular, in a SNIC bifurcation on the upper branch (greatest $p$ values) and SBT occurs on the upper saddle node branch, which coincides locally with the SNIC branch.

The cyan curve (separating (a) and (c), (b) and (g)) corresponds to the disappearance of both folds $\mathrm{FH}_{1}$ and $\mathrm{FH}_{2}$ of the Hopf bifurcation branch. Note that it is not a codimension 3 bifurcation because the bifurcation diagrams (b) for $C \in\left[C_{\mathrm{FH}_{1}}, C_{\mathrm{Cusp}}\right]$ and $(\mathrm{g})$ are diffeomorphic. However, the existence (for a given $C$ value) of a different number of Hopf bifurcations depending on $p$ modifies the structure of the bifurcation diagram according to $p$ and therefore the generated outputs. The transition between ( $g$ ) and (b) thereby inserts an interval of $C$ values for which NITAM-type outputs are generated in the interval of $C$ values generating $\mathrm{NMO}$.

Along the blue curve (separating (c) and (e), (d) and (f)) fusion and separation of the two bifurcations Cusp and Cusp $_{3}$ occurs in the diagram according to $(C, p)$. Starting at (c) (resp. (d)), varying parameters $G$ and $\alpha_{2}$, the two cusp bifurcations occur when $\left(G, \alpha_{2}\right)$ intersects this blue curve, thereby creating in (e) (resp. (f)) an interval of $C$ values for which there is no longer a saddle node bifurcation according to $p$. In this way, this interval where the model generates NMO type-outputs (yellow) merges into the interval where there are NIS-STO type outputs.

The green curves (separating (c) and (d), (e) and (f), (g) and (h), (h) and (i) among other region couples) correspond to the appearance of at least one additional cusp for a positive $C$ value. In the first case, the cusp bifurcation exists for a negative $C$ value, and when $\left(G, \alpha_{2}\right)$ intersects a green curve, this value $C_{\text {Cusp }_{0}}$ becomes positive (as in diagrams (d), (f), and (i)). In another case, when $\left(G, \alpha_{2}\right)$ intersects a green curve, two cusp bifurcations connected by the two same saddle node branches appear as in the transitions from (g) to $(\mathrm{h})$. It is therefore a codimension 3 cusp-cusp bifurcation. 


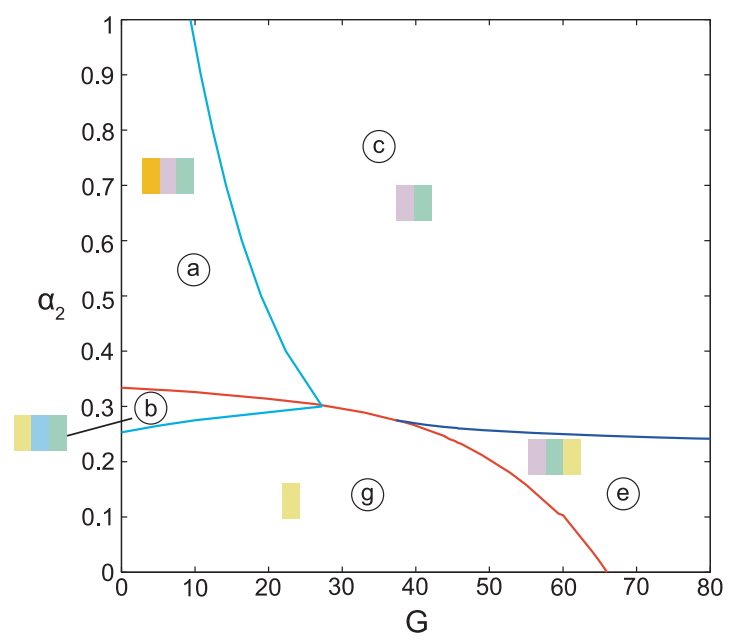

Figure 11: Partition of parameter $\left(\alpha_{2}, G\right)$ space based on the time series panel that the system can generate for $C \in[0,400]$. The cyan curve represents the appearance and disappearance of two folds of the Hopf bifurcation branch. The red curve is a branch of degenerate Bogdanov-Takens bifurcations. The blue curve is a branch of cusp/cusp bifurcation. This diagram defines five regions characterized by a single panel of output types: (a) NIS-OTO, NIS, NIS-STO; (b) NMO, NITAM, NIS-STO; (c) NIS, NIS-STO; (e) NIS, NIS-STO, NMO; (g) NMO.

Transitions between regions that are not adjacent can occur when passing through an intersection point between two curves. Note specifically that the intersection between the red curve and the blue curve is a codimension 4 bifurcation in parameters $G, \alpha_{2}, C$, and $p$ where a degenerate Bogdanov-Takens bifurcation involving a cusp bifurcation coincides with a cusp-cusp bifurcation. Similarly, the intersection point between the red and cyan curves corresponds to simultaneous occurrences of a degenerate Bogdanov-Takens bifurcation and the fusion of two folds of the Hopf bifurcation branch. These two central points in the rectangle partition with values of $\left(G, \alpha_{2}\right)$ primarily organize the time-series panel that the system can generate based on the values given to the direct and indirect excitatory feedback gains.

\subsection{Tool for Estimating Relative Contributions of Direct and Indirect} Excitatory Feedbacks. Certain groups of partition regions in Figure 8 cannot be differentiated by the panel of time series types that the system can generate. This is due to the fact that certain bifurcation diagrams for (a) to (i) differ only in the bifurcations that affect trivial cases. We simplify the partition in Figure 8 by ignoring the appearances of cusp bifurcations along the green curves and obtain the simplified partition in Figure 11. Hence, each 
new region is characterized by a single panel of time series generated for various values of $C \in[0,400]$. This panel is identified with a flag composed of the colors related to the types of time series.

Since diagrams (c) and (d) in Figure 9 differ only by the presence of Cusp $_{0}$, which affects only the trivial cases, regions (c) and (d) are combined into a single region called (c). For $\left(G, \alpha_{2}\right)$ in this new region, the model can only generate NIS and NIS-STO type outputs according to the value of $C$ (mauve and green flag). The same simplification is applied to regions (e) and (f), combined into a single region, (e), in which the time series are of the type NIS, NIS-STO, and NMO (mauve, green, and yellow flag). Regions (g), (h), and (i) are combined in the new region, (g), in which the time series are all of type NMO (yellow flag). Regions (a) and (b) remain unchanged and are characterized by the NIS-OTO, NIS, NIS-STO panels (orange, mauve and green flag) and NMO, NITAM, NIS-STO (yellow, blue and green flag), respectively.

Note that the bifurcation analysis also provides a bijective mapping between the set of time series types and a partition in the extended parameter space $\left(C, G, \alpha_{2}\right)$ that consists of five regions intertwined in a complex way. Yet the partition in the $\left(G, \alpha_{2}\right)$ plane allows us to study the impact of the balance between direct and indirect excitatory feedbacks on the model behavior, assuming that the number of synaptic connections between populations remains constant.

4.4 Comparison with Experimental Data. Neural mass models are classically used to simulate typical features in epileptic seizures (Wendling et al., 2002; Touboul et al., 2011) and more recently to search mechanisms underlying epileptic activities in animal models (Wendling, Bartolomei, Mina, Huneau, \& Benquet, 2012; Huneau et al., 2013). In this regard, we have reproduced experimental data recorded from epileptic mice with our model by tuning the coupling gain of the indirect excitatory feedback. The experimental data were recorded using the mesial temporal lobe epilepsy (MTLE) mouse model (Riban et al., 2002). Mice were initially injected with kainic acid (KA) in the hippocampus and were implanted with intracranial electrodes. After the animals reached the chronic epileptic stage (about 30 days after KA injection), LFP was recorded in the injured hippocampus (2048 Hz sampling frequency). Before each recording, mice were injected with picrotoxin (intraperitoneal). The experimental procedures are detailed in Huneau et al. (2013) and agree with the European Communities Council Directive of November 24, 1986 (86/609/EEC).

The acquired signals contain many hippocampal discharges (HD). HD display high-amplitude sharp waves followed by lower-amplitude rhythmic discharges (Heinrich et al., 2011). Actually two of the behaviors described in section 3 share common features with these HD (see Figure 12a). The NIS case generates sparse large-amplitude spikes comparable with the 
(a) Real data
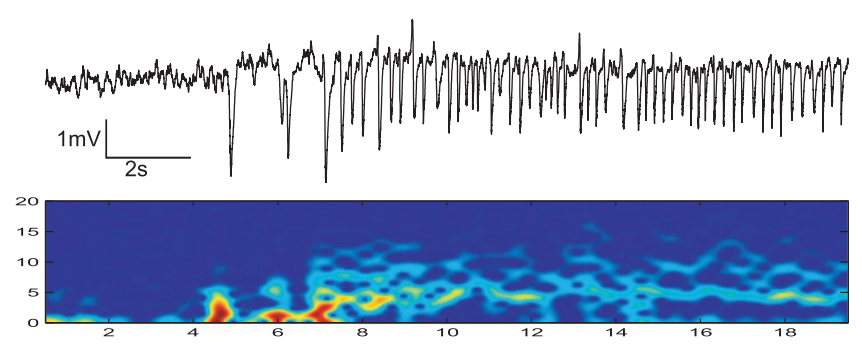

(b) Simulated data
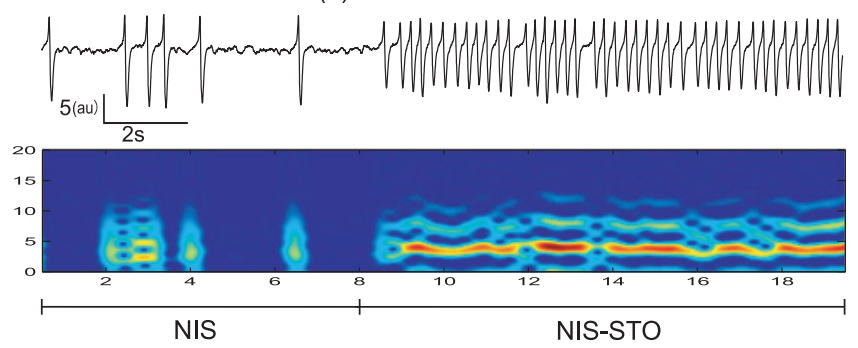

Figure 12: (a) Experimental LFP time series and associated spectrogram. (b) Model output and associated spectrogram. For $t<8 \mathrm{~s}$, NIS case $\left(\alpha_{2}=0.4\right)$ and for $t>8 \mathrm{~s}$, NIS-STO case $\left(\alpha_{2}=0.35\right)$.

first regime, and the NIS-STO case generates lower-amplitude and higherfrequency oscillations comparable with the rhythmic discharges.

Using our model, we have generated a time series displaying NIS behavior for $t<8 s$ and NIS-STO behavior afterward (see Figure 12b). The transition from the NIS case to the NIS-STO case is obtained by switching the $\alpha_{2}$ value (from 0.4 to 0.35 ) at time $t=8 \mathrm{~s}$. When the activity in a given cortical area is changed, the activity in the other areas is also modified, and their feedback action on the considered population also changed; thus, the expectation of $p(t)$ is modified at $t=8 \mathrm{~s}$. The spectrograms associated with the model output and the experimental data (see Figure 12) show that the model output is comparable to the real data in terms of frequency and oscillation amplitudes in each regime. (See Garnier, Huneau, Vidal, Wendling, \& Benali, 2014, for further explanations and an expanded analysis.)

\section{Discussion}

We propose a new neural mass model generalizing existing models. This model, built around a population of pyramidal cells, integrates both types of excitatory feedback present in literature: first, a direct feedback loop of the pyramidal neuron population onto itself (Wilson \& Cowan, 1973; Robinson 
et al., 1997; Liley et al., 2002; Molaee-Ardekani et al., 2010), and second, an indirect feedback produced by the action of pyramidal cells via intermediary pyramidal cells (Jansen et al., 1993; Jansen \& Rit, 1995; Wendling et al., 2000, 2002). To the best of our knowledge, these two types of excitatory coupling have never been studied together within a same model.

This architecture allows us to study the balance between direct (via parameter $G$ ) and indirect (via parameter $\alpha_{2}$ ) excitatory feedbacks on the dynamical behavior of the model. We have characterized the codimension 2 bifurcations undergone by the system for $(C, p) \in[0,400] \times[0,2000]$ based on the different values of $\left(\alpha_{2}, G\right)$ in the rectangle $[0,1] \times[0,80]$. This study has revealed a rich zoology of dynamical behaviors that are physiologically relevant and identified.

This study highlights another fact about neural mass models based on the classic second-order operators $\mathcal{F}_{e}$ and $\mathcal{F}_{i}$ for the synaptic connection kinetics: such a model involving only direct excitatory feedback cannot reproduce the whole set of behaviors identified in this letter when considering physiological parameter values. This neural mass model, obtained from our model by setting $\alpha_{2}=0$, cannot produce time series of the NISOTO or NITAM type. Moreover, a Jansen-Rit-like model considering an indirect feedback ( $G=0$ in our model) displays NIS-STO-type behaviors for reasonable values of $C$ (lower than 200) only in a small range of $\alpha_{2}$ (precisely ]0.31, 0.34[). Nevertheless, it is true that this result remains limited by the parameter space studied. It would be interesting to look at a largerparameter space of higher dimension in which other bifurcations would be likely to occur. In fact, for $G>66$ and high $p(p>2000)$ Figures $9 \mathrm{c}$ and $9 \mathrm{e}$ reveal a Bogdanov-Takens bifurcation on the upper branch of the Hopf bifurcations, which produces two saddle node bifurcation branches replacing the Hopf bifurcations. In this case, the cusp bifurcations fuse for a certain $G$ value, provoking the disappearance of a branch of Hopf bifurcations in the long term. Although it seems far from the physiological values of parameters observed in the literature, this behavior could be interesting to analyze from the dynamical viewpoint in order to improve our understanding of the model. To go even further, it would be interesting to expand this study to other parameters, notably concerning the synaptic responses, which are critical to the dynamical behaviors of this type of models (Grimbert \& Faugeras, 2006).

Studying the proposed model allows us to define an exhaustive glossary (within the studied parameter space) of time series types, physiologically realistic, generated by this model for different values of $\alpha_{2}, G$, and $C$. The creation of this glossary consists in resolving the direct problem, which associates parameter values with types of dynamics. In this way, this partition would enable a qualitative resolution of the inverse problem concerning the estimation of direct and indirect coupling gain parameters from time series organization and identify their distribution in the parameter space, assuming that the number of synaptic connections $C$ between populations remains 
constant. For example, in a set of NMO and NIS time series, the partition shown in Figure 11 allows us to determine that the values of direct and indirect excitatory coupling gains are located in region (e), the only region where the model can generate these types of outputs. This estimating method can be fine-tuned when the NIS-STO are being studied. In fact, such outputs can occur in all presented codimension 2 bifurcation diagrams according to $p$ and $C$, even in region $(\mathrm{g})$. However, within this region, the set of $\left(G, \alpha_{2}\right)$ values for which NIS-STO occurs is a very small neighborhood of the red degenerate Bogdanov-Takens bifurcation curve, which we have chosen not to show in Figures 8 and 11. For $\left(G, \alpha_{2}\right)$ in the main part of this region, there is a cusp bifurcation in the bifurcation diagram according to $(C, p)$ (indicated with an arrow in the panels in Figure 10) for a very high $C$ value and therefore far from physiological reality. However, for $\left(G, \alpha_{2}\right)$ in region (g) but close to the red curve, this cusp bifurcation occurs for $C$ values in the $[0,400]$ interval being studied. The occurrence of NIS-STO and NMO-type outputs without any other type allows us to infer the region of possible values for $\left(G, \alpha_{2}\right)$ with greater accuracy and improve the method for qualitatively solving the inverse problem of estimating parameters. When experimental time series are used, this analysis could be used for a model-based estimation of coupling gains parameters between populations. Other works on this subject have previously addressed this identification parameter problem by building a space representing time series based on parameters (Wendling et al., 2002; Labyt, Frogerais, Uva, Bellanger, \& Wendling, 2007). However, these studies are proceeding empirically through repeated simulations. In this study, we have built a theoretical diagram using the bifurcation analysis, which has enabled a more precise and exhaustive partition.

Touboul et al. (2011) have studied bifurcations in the Jansen-Rit model. Our study has allowed us to identify the additional dynamical behaviors generated by NIS-STO and NITAM type outputs. The proximity of this output, within the parameter space, with other more traditional behaviors such as NIS gives them definite physiological relevance. However, to the best of our knowledge, these behaviors and associated times series have never been specifically described in electrophysiological signals. We would argue that the reason is they have never been researched. In addition, the associated time series can easily be interpreted as a noise even though they are strictly separated from the dynamical viewpoint. Therefore, the proposed study can have a predictive value and serve as a tool for researching subtle dynamical behaviors in physiological or even pathological cases.

\section{Appendix: Bifurcations and Numerical Methods}

We recall briefly the properties of the different types of bifurcations undergone by the model and identified in this letter. We describe the numerical 
methods used for calculating the bifurcation diagrams in Figures 3 to 7 and 9 and 10 and for the construction of the partition of $\left(G, \alpha_{2}\right)$ space in Figure 8 .

We have implemented these methods on a Matlab platform. We took advantage of the explicit characterizations of the singular points as well as the saddle node, cusp, and cusp/cusp bifurcations. The localizations of the limit cycle families and bifurcations are based on pseudo-arclength continuation methods (see Doedel, Govaerts, \& Kuznetsov, 2003; Govaerts, Kuznetsov, \& Dhooge, 2005; Kuznetsov, Govaerts, Doedel, \& Dhooge, 2005). Few transitions between dynamical organizations are not accessible to continuation-based algorithms since they do not correspond to generic bifurcations, as explained in section 4.1. We have integrated an automatic research of such transitions in our algorithms. With these codes, we were able to produce automatically the codimension 2 bifurcation diagrams, displayed in Figure 9 and 10, provided that the values of the parameters associated with the research algorithms (e.g., arc length step, tolerances, initialization, interval of model parameter values) were well chosen in each case. Hence, the design of a completely automatic algorithm for building the transition curves in Figure 8 is delicate yet conceivable. For efficiency, the transition curves (except the cusp/cusp bifurcation curve, which is characterized by an explicit condition) have been built by a humandirected process based on the analysis of the codimension 2 bifurcation diagram.

A.1 Singular Points and Limit Cycles. A direct calculation shows that any singular point of system 2.3 with constant input $p(t)=p$ is characterized by its $y_{0}$ component as a fixed point of the following function $\varphi$ :

$$
\begin{aligned}
\varphi\left(y_{0}\right)= & \frac{A}{a} \operatorname{sigm}\left(\frac{A}{a}\left(\alpha_{2} C \operatorname{sigm}\left(\alpha_{1} C y_{0}\right)+p\right)+G y_{0}\right. \\
& \left.-\frac{B}{b} \alpha_{4} C \operatorname{sigm}\left(\alpha_{3} C y_{0}\right), e_{0}, r, v_{0}\right) .
\end{aligned}
$$

The other components of the singular point associated with such value $y_{0}$ are given by

$$
\begin{aligned}
& y_{1}=\frac{A}{a}\left(\alpha_{2} C \operatorname{sigm}\left(\alpha_{1} C y_{0}\right)+p\right)+G y_{0} \\
& y_{2}=\frac{B}{b} \alpha_{4} C \operatorname{sigm}\left(\alpha_{3} C y_{0}\right) \\
& y_{3}=y_{4}=y_{5}=0
\end{aligned}
$$


One can state an equivalent characterization if we restrict $y_{0}$ values to ]0, $2 A e_{0} / a[$. This interval of values defines an invariant domain in the phase space for the forward flow. Hence, we use the following property: for any $\left.y_{0} \in\right] 0,2 A e_{0} / a_{1}\left[\right.$, there exists a unique value of $p$ such that $\left(y_{i}\right)_{i=0}^{5}$ defined by equation A.2, is a singular point of equation 2.3 and given by the following explicit formula:

$$
\begin{aligned}
p=\psi\left(y_{0}\right)= & \frac{a}{A}\left(v_{0}-\frac{1}{r} \ln \left(\frac{2 A e_{0}}{a y_{0}}-1\right)-G y_{0}+\frac{B}{b} \alpha_{4} C \operatorname{sigm}\left(\alpha_{3} C y_{0}\right)\right) \\
& -\alpha_{2} C \operatorname{sigm}\left(\alpha_{1} C y_{0}\right) .
\end{aligned}
$$

The stability of a given singular point $Q=\left(y_{i}\right)_{i=0}^{5}$ is characterized by the linearized flow of equation 2.3, at this point, that is, by the eigenvalues of the following Jacobian matrix evaluated at $Q$ :

$$
J(Q)=\left(\begin{array}{ll}
0_{3} & I_{3} \\
U & V
\end{array}\right) \in \mathcal{M}_{6}(\mathbb{R})
$$

where $0_{3} \in \mathcal{M}_{3}(\mathbb{R})$ is the null matrix, $I_{3} \in \mathcal{M}_{3}(\mathbb{R})$ is the identity matrix,

$$
\begin{aligned}
U & =\left(\begin{array}{ccc}
-a^{2} & \Theta & -\Theta \\
A a \alpha_{1} \alpha_{2} C^{2} S\left(\alpha_{1} C y_{0}\right) & G \Theta-a^{2} & -G \Theta \\
B b \alpha_{3} \alpha_{4} C^{2} S\left(\alpha_{3} C y_{0}\right) & 0 & -b^{2}
\end{array}\right), \\
V & =\left(\begin{array}{ccc}
-2 a & 0 & 0 \\
0 & -2 a & 0 \\
0 & 0 & -2 b
\end{array}\right) \\
S(v) & =\operatorname{sigm}^{\prime}(v)=2 e_{0} r \frac{e^{r\left(v_{0}-v\right)}}{\left(1+e^{r\left(v_{0}-v\right)}\right)^{2}}, \\
\Theta & =\operatorname{AaS}\left(y_{1}-y_{2}\right) .
\end{aligned}
$$

A singular point $Q$ is hyperbolic if every eigenvalue of $J(Q)$ has a nonzero real part. A hyperbolic singular point $Q$ is stable if every eigenvalue of $J(Q)$ has a negative real part and unstable otherwise. For simplicity, we refer to "the eigenvalues of $Q$ " as the eigenvalues of the Jacobian matrix associated with the flow at $Q$.

The branch of singular points in Figures 3 to 7 are obtained using equation A.3 and the stability of the singular points by numerical evaluation of the associated eigenvalues. 


\section{A.2 Codimension 1 Bifurcation of Singular Points.}

A.2.1 Saddle Node Bifurcation. A saddle node bifurcation is a collision and disappearance of two singular points occurring when the bifurcation parameter varies. Near the bifurcation value, the two singular points have $k$ and $k+1$ associated real negative eigenvalues, respectively. For system 2.3 , each saddle node bifurcation according to parameter $p$ corresponds to a fold of the curve of singular points defined by equation A.3, that is, by equation $\psi^{\prime}\left(y_{0}\right)=0$, where

$$
\begin{aligned}
\psi^{\prime}\left(y_{0}\right)= & \frac{2 a e_{0}}{\left(2 A e_{0}-a y_{0}\right) y_{0} r}-\frac{a G}{A}+\frac{a B \alpha_{3} \alpha_{4} C^{2} \operatorname{sigm}^{\prime}\left(\alpha_{3} C y_{0}\right)}{A b} \\
& -\alpha_{1} \alpha_{2} C^{2} \operatorname{sigm}^{\prime}\left(\alpha_{1} C y_{0}\right)
\end{aligned}
$$

The corresponding value of $p$ is given by equation A.3. The branches of saddle node bifurcations in Figure 9 are obtained by solving, for each point of a given subdivision of the interval of $C$ values, $\psi^{\prime}\left(y_{0}\right)=0$ in $y_{0}$ and evaluating $p=\psi\left(y_{0}\right)$ afterward for each solution $y_{0}$. Transversality conditions (see Guckenheimer \& Holmes, 1993) on the flow are checked afterward to ensure the nondegeneracy of the bifurcation.

A.2.2 Hopf Bifurcation. A Hopf bifurcation is the local birth of a limit cycle from a singular point when it changes stability by a pair of purely imaginary eigenvalues. The bifurcation is said to be supercritical (resp. subcritical) if the cycle is stable (resp. unstable). More precisely, let us assume that for each value $p \in\left[p_{H}-\delta p, p_{H}+\delta p\right]$, system 2.3 admits a singular point $Y(p)$. The singular point $Y(p)$ undergoes a Hopf bifurcation at $p=p_{H}$ if a couple of eigenvalues $\lambda(p) \pm i \mu(p)$ associated with $Y(p)$ cross the imaginary axis at $p=p_{H}$. To fix the idea, let us assume that $\lambda(p)<0$ for $p \in\left[p_{H}-\delta p, p_{H}[\right.$ and $\lambda(p)>0$ for $p \in] p_{H}, p_{H}+\delta p$ ]. If the Hopf bifurcation is supercritical (resp. subcritical), a family of stable (resp. unstable) limit cycles indexed by $\left.p \in] p_{H}, p_{H}+\delta p\right]$ (resp. $p \in\left[p_{H}-\delta p, p_{H}\left[\right.\right.$ ) emerges from $Y\left(p_{H}\right)$. Following (Kuznetsov, 2004), whether a Hopf bifurcation is supercritical or subcritical is determined by the sign of the first Lyapunov exponent of the system near the singular point.

Numerically, Hopf bifurcations are located by a change of sign in the real part of a couple of complex conjugate eigenvalues associated with a singular point. Since equation A.3 provides an explicit characterization of the singular points, a direct calculation of the eigenvalues on a grid of $(C, p)$ values is more efficient than in the general case. For each point $(C, p)$ corresponding to Hopf bifurcation, the first Lyapunov exponent is calculated following Kuznetsov (2004). 


\section{A.3 Limit Cycles and Codimension One Bifurcations.}

A.3.1 Pseudo-arc-length Continuation of Limit Cycles. The families of limit cycles shown in Figures 3 to 7 are obtained using a pseudo-arc-length continuation method. Numerical continuation methods consist in reducing the search for a family of limit cycles indexed by a parameter to the sequential search for the root of functions built on a discretization of the flow and a periodic boundary condition. Introducing a pseudo-arc-length condition instead of fixing the grid of index parameter values makes it possible to keep tracking the limit cycles even if the family of limit cycles displays a fold. Hence, this algorithmic process allows us to track branches of stable as well as unstable limit cycles. (For more details about this method, see Doedel et al., 2003, and Govaerts et al., 2005.)

For building the families of limit cycles of system 2.3, we have characterized each cycle by 1000 to 2000 mesh points (depending on the maximal amplitude and period along the branch of limit cycles), and we used the collocation points associated with the fourth-order Lobatto IIIa method.

A.3.2 Fold Bifurcation of Limit Cycles. A fold bifurcation of limit cycles corresponds to a turning point of a branch of limit cycles. Hence, such bifurcation undergone by system 2.3 according to $p$ can be directly located from the family of limit cycles obtained with the method described above. For building the branches of fold bifurcations of limit cycles in Figure 9, we have used the arc-length continuation method with the additional equation expressing the tangential condition (local existence of two roots) and considering $C$ as a variable in the arc-length continuation equation (see Kuznetsov et al., 2005).

\section{A.4 Codimension 2 Bifurcations.}

A.4.1 Cusp of Singular Points. A cusp bifurcation occurs when a singular point has one zero eigenvalue and the quadratic coefficient for the saddle node bifurcation vanishes. At the cusp bifurcation point, two branches of saddle node bifurcation meet tangentially and disappear. For nearby parameter values, the system can have three singular points that collide and disappear pairwise via the saddle node bifurcations.

Following the characterization of saddle node bifurcation given by equations A.3 and A.4, cusp bifurcations are defined as the points in this set of solutions fulfilling $\psi^{\prime \prime}\left(y_{0}\right)=0$ and $\psi^{(3)}\left(y_{0}\right) \neq 0$.

A.4.2 Bogdanov-Takens Bifurcation. A Bogdanov-Takens occurs when a singular point has a zero eigenvalue of multiplicity two. For nearby parameter values, the system admits a saddle and a nonsaddle singular point that collide and disappear via a saddle node bifurcation. The nonsaddle singular point undergoes a Hopf bifurcation giving birth to a limit cycle. This cycle 
persists for nearby parameter values until it disappears via a homoclinic bifurcation. Hence, a Bogdanov-Takens bifurcation occurs at a point of a saddle node bifurcation branch. From this point arise, both tangentially to the saddle node bifurcation branch, a branch of Hopf bifurcations and a branch of homoclinic bifurcations. Whether it is subcritical or supercritical depends on the nature of the Hopf bifurcation nearby the Bogdanov-Takens bifurcation point and, consequently, on the first Lyapunov exponent.

Numerically we find the Bogdanov-Takens bifurcation point by searching for the vanishing of a second eigenvalue associated with the nonhyperbolic point along the branch of saddle node bifurcations. We check the nondegeneracy condition (see Guckenheimer \& Holmes, 1993) involving the quadratic coefficients in the Taylor expansion of the flow at the bifurcation point, which also determines whether the bifurcation is subcritical or supercritical.

A.4.3 Bautin Bifurcation. A Bautin (or generalized Hopf) bifurcation separates branches of subcritical and supercritical Hopf bifurcations in a twoparameter plane. For nearby parameter values, the system admits two limit cycles that collide and disappear via a fold bifurcation of limit cycles. It is characterized by a pair of purely imaginary eigenvalues associated with the singular point and a vanishing first Lyapunov coefficient for the Hopf bifurcation.

Since we locate the branches of Hopf bifurcations in the bifurcation diagrams according to $C$ and $p$ and calculate the first Lyapunov coefficient at each point, Bautin bifurcation points in Figure 9 and 10 are obtained directly. We just check the nondegeneracy conditions (see Kuznetsov, 2004).

A.4.4 Fold Points of the Hopf Bifurcation Branch. As explained in section 4.1, the fold points of a branch of Hopf bifurcations do not correspond to codimension 2 bifurcations but participate in the organization of the bifurcation diagram according to $p$ and the associated time series. They are given directly by the numerical calculation of the Hopf bifurcation branches.

A.5 Partition of $\left(G, \alpha_{2}\right)$ Rectangle. Branches defining the partition of the rectangle $[0,80] \times[0,1]$ of $\left(G, \alpha_{2}\right)$ values are obtained in two ways. The branch involving the appearance of cusps (AC and cusp/cusp) is obtained using the numerical tracking of cusp bifurcations described above. The branches of the appearance of folds in the branch of Hopf bifurcation $(\mathrm{FH} / \mathrm{FH})$ and degenerate Bogdanov-Takens (DBT) bifurcation are calculated using dichotomic processes.

A.5.1 AC and Cusp/Cusp Branches. The AC branch consists in the appearance of cusp bifurcations in the bifurcation diagram according to $C$ and $p$ either by a cusp/cusp bifurcation or by the passage of a cusp bifurcation from negative to positive values of $C$. As explained above, cusp bifurcations 
are characterized by the existence of a value $y_{0}$ fulfilling

$$
\begin{aligned}
\psi\left(y_{0}\right) & =p, \\
\psi^{\prime}\left(y_{0}\right) & =0, \\
\psi^{\prime \prime}\left(y_{0}\right) & =0, \\
\psi^{(3)}\left(y_{0}\right) & \neq 0 .
\end{aligned}
$$

Note that in the case of a cusp/cusp bifurcation, the fourth condition fails and should be replaced by the vanishing $\psi^{(3)}\left(y_{0}\right)$ together with other nondegeneracy conditions. Since different cusp bifurcations are involved $\left(\mathrm{Cusp}_{0}\right.$ to $\left.\mathrm{Cusp}_{3}\right)$ and can be quickly numerically determined by solving system A.5, we have chosen to directly calculate the cusps bifurcations and find where they collide or enter $C>0$. We have first approximated the AC and cusp/cusp branches by considering a coarse grid of the rectangle with $10^{-2}$ step for $\alpha_{2}$ and 1 step for $G$. Then we have obtained more precise values for the mesh points of the branches by applying a dichotomic search according to $\alpha_{2}$ for the cusp/cusp points and to $G$ for the AC points.

A.5.2 DBT and FH/FH Branches. The degenerate Bogdanov-Takens (also known as Dumortier-Roussarie-Sotomayor) bifurcation is a codimension 3 bifurcation corresponding to the occurrence of a Bogdanov-Takens bifurcation at a cusp point (see Dumortier et al., 1991). The cusp bifurcations have been located on a coarse grid of $\left(\alpha_{2}, G\right)$ values to approximate the AC and cusp/cusp branches. Hence, we locate the DBT bifurcations using a dichotomic process according to parameter $\alpha_{2}$ for each value of $G$ of the coarse grid that calculates the Hopf bifurcation branch and determines the value of $\left(G, \alpha_{2}\right)$ for which a cusp point crosses the Hopf bifurcation branch.

The FH/FH branch corresponds to the appearance of folds in the branch of Hopf bifurcation. We locate this branch with a dichotomic process according to parameter $G$ values that calculate the Hopf bifurcation branch and determine whether it displays a fold.

\section{Acknowledgments}

This work was performed within the Labex SMART (ANR-11-LABX-65) supported by French state funds managed by the ANR within the Investissements d'Avenir program under reference ANR-11-IDEX-0004-02. C.H. holds a grant from Fondation Leducq Transatlantic Network of Excellence on pathogenesis of small vessel disease of the brain. We thank Fabrice Wendling for allowing us to use real data acquired at LTSI-UMR Inserm 1099, Rennes. We also thank Fabrice Wendling and Jean-Pierre Françoise for interesting discussions and Mélanie Pelegreni-Issac for her manuscript revision. 


\section{References}

Baer, S., Kooi, B., Kuznetsov, Y., \& Thieme, H. (2006). Multiparametric bifurcation analysis of a basic two-stage population model. SIAM I. Appl. Math., 66(4), 13391365.

Beurle, R. (1956). Properties of a mass of cells capable of regenerating pulses. $\underline{\text { Phil. }}$ Trans. R. Soc. B, 240(669), 55-94.

Braitenberg, V., \& Schüz, A. (1991). Anatomy of the cortex: Statistics and geometry. New York: Springer.

Brunel, N., \& Wang, X. J. (2001). Effects of neuromodulation in a cortical network model of object working memory dominated by recurrent inhibition. Iournal. Comput. Neurosci., 11(1), 63-85.

Dayan, P., \& Abbott, L. F. (2001). Theoretical neuroscience: Computational and mathematical modeling of neural systems. Cambridge, MA: MIT Press.

Deco, G., Jirsa, V. K., Robinson, P. A., Breakspear, M., \& Friston, K. (2008). The dynamic brain: From spiking neurons to neural masses and cortical fields. $\underline{P L O S}$ Comput. Biol., 4(8), e1000092.

Doedel, E. J., Govaerts, W., \& Kuznetsov, Yu. A. (2003). Computation of periodic solution bifurcations in ODEs using bordered systems. SIAM I. Numer. Anal., 41(2), 401-435.

Dumortier, F., Roussarie, R., Sotomayor, J., \& Zoladek, H. (1991). Bifurcation of planar vector fields. Lect. Notes Math., 1480, 165-224.

Freeman, W. (1975). Mass action in the nervous system. New York: Academic Press.

Frick, A., Feldmeyer, D., Helmstaedter, M., \& Sakmann, B. (2008). Monosynaptic connections between pairs of L5A pyramidal neurons in columns of juvenile rat somatosensory cortex. Cereb. Cortex, 18(2), 397-406.

Garnier, A., Huneau, C., Vidal, A., Wendling, F., \& Benali, H. (2014). Identification of dynamical behaviors in epileptic discharges using a neural mass model with double excitatory feedbacks. In Proceedings of ICCSA (pp. 205-210). url:litis.univ-lehavre.fr/ bertelle/iccsa2014/iccsa2014proceedings.pdf

Gerstner, W., \& Kistler, W. M. (2002). Mathematical formulations of Hebbian learning. Biol. Cubern., 87(5-6), 404-415.

Govaerts, W., Kuznetsov, Y. A., \& Dhooge, A. (2005). Computation of periodic solution bifurcations in ODEs using bordered systems. SIAM I. Sci. Comput., 27(1), 231-252.

Griffith, J. (1963). A field theory of neural nets I: Derivation of field equations. Bull. Math. Biol., 25, 111-120.

Griffith, J. (1965). A field theory of neural nets II: Properties of the field equations. Bull. Math. Biol., 27, 187-195.

Grimbert, F., \& Faugeras, O. (2006). Bifurcation analysis of Jansen's neural mass model. Neural Comput., 18(12), 3052-3068.

Guckenheimer, J., \& Holmes, P. (1993). Nonlinear oscillations, dynamical systems and bifurcations of vector fields. New York: Springer.

Heinrich, C., Lähteinen, S., Suzuki, F., Anne-Marie, L., Huber, S., Häussler, U., . . Depaulis, A. (2011). Increase in BDNF-mediated TrkB signaling promotes epileptogenesis in a mouse model of mesial temporal lobe epilepsy. Neurobiol. Dis., $42(1), 35-47$. 
Hodgkin, A. L., \& Huxley, A. F. (1952). A quantitative description of membrane current and its application to conduction and excitation in nerve. L. Physiol., $117(4), 500-544$.

Huneau, C., Benquet, P., Dieuset, G., Biraben, A., Martin, B., \& Wendling, F. (2013). Shape features of epileptic spikes are a marker of epileptogenesis in mice. Epilepsia, 54(12), 2219-2227.

Jansen, B. H., \& Rit, V. G. (1995). Electroencephalogram and visual evoked potential generation in a mathematical model of coupled cortical columns. Biol. Cybern., 73(4), 357-366.

Jansen, B. H., Zouridakis, G., \& Brandt, M. E. (1993). A neurophysiologically-based mathematical model of flash visual evoked potentials. Biol. Cubern., 68(3), 275283.

Jirsa, V. K., \& Haken, H. (1996). Field theory of electromagnetic brain activity. Phys. Rev. Lett., 77(5), 960-963.

Jirsa, V. K., \& Haken, H. (1997). A derivation of a macroscopic field theory of the brain from the quasi-microscopic neural dynamics. Physica D, 99(4), 503-526.

Katznelson, R. D. (1981). Normal modes of the brain: Neuroanatomical basis and a physiological theoretical model. In P. Nunez (Ed.), Electrical fields of the brain: The neurophysics of EEG. (pp. 401-442). New York: Oxford University Press.

Knight, B., Manin, D., \& Sirovich, L. (1996). Dynamical models of interacting neuron populations. In E. C. Gerf (Ed.), Symposium on Robotics and Cybernetics: Computational Engineering in Systems Applications. Lille, France: Cite Scientifique.

Kuznetsov, Y. (2004). Elements of applied bifurcation theory (3rd ed.). New York: Springer.

Kuznetsov, Y. A., Govaerts, W., Doedel, E. J., \& Dhooge, A. (2005). Numerical periodic normalization for codim 1 bifurcations of limit cycles. SIAM I. Numer. Anal., 43(4), 1407-1435.

Labyt, E., Frogerais, P., Uva, L., Bellanger, J.-J., \& Wendling, F. (2007). Modeling of entorhinal cortex and simulation of epileptic activity: Insights into the role of inhibition-related parameters. IEEE Inform. Technol. B, 11, 450-461.

Liley, D.T.J., Cadusch, P. J., \& Dafilis, M. P. (2002). A spatially continuous mean field theory of electrocortical activity. Network: Comput. Neural Syst., 13(1), 67-113.

Liley, D.T.J., \& Walsch, M. (2013). The mesoscopic modeling of burst suppression during anesthesia. Front. Comput. Neurosci., 7(46).

Lopes da Silva, F. H., Hoeks, A., Smits, H., \& Zetterberg, L. H. (1974). Model of brain rythmic activity. Kubernetik, 15(1), 27-37.

Lopes da Silva, F. H., van Rotterdam, A., Barts, P., van Heusden, E., \& Burr, W. (1976). Models of neuronal populations: The basic mechanisms of rhythmicity. Prog. Brain. Res., 45, 281-308.

McKinney, R. A., Debanne, D., Gähwiler, B. H., \& Thompson, S. M. (1997). Lesioninduced axonal sprouting and hyperexcitability in the hippocampus in vitro: Implications for the genesis of post-traumatic epilepsy. Nat. Med., 3, 990-996.

Miles, R., \& Wong, R. K. (1986). Excitatory synaptic interactions between CA3 neurones in the guinea-pig hippocampus. I. Phusiol., 373, 397-418.

Molaee-Ardekani, B., Benquet, P., Bartolomei, F., \& Wendling, F. (2010). Computational modeling of high-frequency oscillations at the onset of neocortical partial seizures: From "altered structure" to "dysfunction". NeuroImage, 52(3), 1109-1122. 
Omurtag, A., Knight, B., \& Sirovich, L. (2000). On the simulation of large populations of neurons. I. Comput. Neurosci., 8, 51-53.

Riban, V., Bouilleret, V., Pham-Lê, B. T., Fritschy, J.-M., Marescaux, C., \& Depaulis, A. (2002). Evolution of hippocampal epileptic activity during the development of hippocampal sclerosis in a mouse model of temporal lobe epilepsy. Neuroscience, 112(1), 101-111.

Robinson, P., Rennie, C., \& Wright, J. (1997). Propagation and stability of waves of electrical activity in the cerebral cortex. Phys. Rev. E, 56(1), 826-840.

Salin, P., Tseng, G. F., Hoffman, S., Parada, I., \& Prince, D. A. (1995). Axonal sprouting in layer $\mathrm{V}$ pyramidal neurons of chronically injured cerebral cortex. J. Neurosci., $15,8234-8245$.

Stefanescu, R. A., \& Jirsa, V. K. (2008). A low dimensional description of globally coupled heterogeneous neural networks of excitatory and inhibitory neurons. PLoS Comput. Biol., 4(11), e1000219.

Suffczynski, P., Kalitzin, S., \& Lopes da Silva, F. H. (2004). Dynamics of nonconvulsive epileptic phenomena modeled by a bistable neural network. Neuroscience, 126(2), 467-484.

Touboul, J., Wendling, F., Chauvel, P., \& Faugeras, O. (2011). Neural mass activity, bifurcations, and epilepsy. Neural Comput., 23(12), 3232-3286.

Van Rotterdam, A., Lopes da Silva, F. H., Van den Ende, J., Viergever, M., \& Hermans, A. (1982). A model of the spatial-temporal characteristics of the alpha rhythm. Bull. Math. Biol., 44(2), 283-305.

Wang, Y., Markram, H., Goodman, P. H., Berger, T. K., Ma, J., \& Goldman-Rakic, P. S. (2006). Heterogeneity in the pyramidal network of the medial prefrontal cortex. Nature Neurosci., 9(4), 534-542.

Wendling, F., Bartolomei, F., Bellanger, J.-J., \& Chauvel, P. (2002). Epileptic fast activity can be explained by a model of impaired GABAergic dendritic inhibition. Eur. I. Neurosci., 15(9), 1499-1508.

Wendling, F., Bartolomei, F., Mina, F., Huneau, C., \& Benquet, P. (2012). Interictal spikes, fast ripples and seizures in partial epilepsies-combining multi-level computational models with experimental data. Eur. I. Neurosci., 36(2), 2164-2177.

Wendling, F., Bellanger, J.-J., Bartolomei, F., \& Chauvel, P. (2000). Relevance of nonlinear lumped-parameter models in the analysis of depth-EEG epileptic signals. Biol. Cubern., 83(4), 367-378.

Wilson, H. R., \& Cowan, J. D. (1972). Excitatory and inhibitory interactions in localized populations of model neurons. Biophus. I., 12(1), 1-24.

Wilson, H. R., \& Cowan, J. D. (1973). A mathematical theory of the functional dynamics of cortical and thalamic nervous tissue. Kibernetik, 13(2), 55-80.

Wong, K.-F., \& Wang, X.-J. (2006). A recurrent network mechanism of time integration in perceptual decisions. L. Neurosci., 26(4), 1314-1328.

Received April 30, 2014; accepted August 10, 2014. 